\title{
A search for candidate light echoes: Photometry of supernova environments ${ }^{\star}$
}

\author{
F.R. Boffi, W.B. Sparks, and F.D. Macchetto ${ }^{\star \star}$ \\ Space Telescope Science Institute, 3700 San Martin Drive, Baltimore, MD 21218, U.S.A. \\ e-mail: boffi@stsci.edu, sparks@stsci.edu, macchetto@stsci.edu
}

Received April 19; accepted June 7, 1999

\begin{abstract}
Supernova (SN) light echoes could be a powerful tool for determining distances to galaxies geometrically, Sparks (1994). In this paper we present CCD photometry of the environments of 64 historical supernovae, the first results of a program designed to search for light echoes from these SNe.

We commonly find patches of optical emission at, or close to, the sites of the supernovae. The color distribution of these patches is broad, and generally consistent with stellar population colors, possibly with some reddening. However there are in addition patches with both unusually red and unusually blue colors. We expect light echoes to be blue, and while none of the objects are quite as blue in $V-R$ as the known light echo of SN 1991T, there are features that are unusually blue and we identify these as candidate light echoes for follow-on observations.
\end{abstract}

Key words: scattering - supernovae: general — distance scale

\section{Introduction}

It is of paramount importance to determine distances to external galaxies: such knowledge impacts both stellar and extragalactic astrophysics as well as cosmology and the structure of the Universe including the derivation of the Hubble constant $\left(H_{0}\right)$. Unfortunately the standard "luminosity-calibrated" methods of distance determination have not yet allowed an unambiguous

Send offprint requests to: F.R. Boffi

* Tables $2 \mathrm{a}$ and $2 \mathrm{~b}$ are also available at the CDS via anonymous ftp to cdsarc.u-strasbg.fr (130.79.128.5) or via http://cdsweb.u-strasbg.fr/Abstract.html and Figs. 1 through 36 are only available in the online version of the journal at http://www.edpsciences.com

** Affiliated to the Astrophysics Division of the Space Science Department of ESA. determination of the Hubble constant (see the Proceedings of "The Extragalactic Distance Scale" STScI May 1996 Symposium for an overview), and all luminosity based indicators are extinction and metallicity dependent in some way. Thus (new) physical and geometric methods of distance determination are highly desirable and should be pursued wherever possible.

Sparks (1994) proposed that distances to external galaxies (well in excess of the distance to the Virgo cluster) could be determined by means of supernova (SN) light echoes. The method is appealing as it is purely geometrical, does not need any secondary distance indicators or calibration and might be used to relate to luminosity distance indicators when applied to galaxies hosting Cepheids and/or Type Ia supernovae (Sparks 1994, 1996).

The technique requires high resolution imaging polarization observations of SN light echoes. A light echo is expected to be produced by scattering of the SN light by dust in the interstellar medium, and would be visible to the observer at some time after the supernova explosion (due to light travel time effects). The evolution of such a feature is well understood (e.g. Chevalier 1986) and mathematically straightforward. To summarize, the light echo is a paraboloid at the focus of which the historical supernova lies. The observer looks down the axis, "into" the paraboloid. While the intensity distribution of the echo is likely to be complex, depending primarily on dust density, by contrast, the polarization distribution should be simple. Angular distance from the supernova is related to the scattering angle, and polarization depends simply on scattering angle, maximizing at $90^{\circ}$ scattering. Hence at the intersection between the plane of the sky containing the supernova and the parabola, light is scattered at an angle of 90 degrees and forms a ring in an image of the degree of polarization (this light having the maximum degree of polarization). The linear diameter of the ring is $2 c t$, where $c$ is the speed of light and $t$ is the time since the explosion, therefore, if $\phi$, the angular diameter of the 
ring is measured, the distance $D$ is derived geometrically by $D=2 c t / \phi$.

Light echoes have been observed around SN 1987A (Crotts 1988; Sparks et al. 1989) and SN $1991 \mathrm{~T}$ (Schmidt et al. 1994). From the ground we would not expect to resolve light echoes around known historical supernovae in most cases. Yet from the ground candidate light echoes can be sought and identified by using imaging and, when appropriate, polarization criteria: a good light echo candidate shows optical emission at/near the site of the historical supernova, is blue in color, because of both the intrinsic "blueness" of the SN and the additional blueing introduced by the scattering process, and is polarized. The discovery of light echo candidates and subsequent confirmation either through ground-based spectroscopic observations, or direct space imaging polarimetry observations is an essential step in the process of enabling general geometric galaxy distance determinations by this technique. Here we describe a program to search for candidate echoes via CCD imaging. Sparks et al. (1999) show how space imaging polarimetry can be used in the well known case of the SN 1991T light echo. The region of maximum linear polarization emission cannot be resolved yet due to the small time since the explosion and the distance to the galaxy. However, we give a distance estimate to SN 1991T (an upper limit of $15 \mathrm{Mpc}$ is found) via simple modeling of the scattering process.

Although the main objective of such a search program is to find light echoes and use them to determine distances, the photometric and spectroscopic data collected, together with the polarimetry, should also provide important information on the supernova environments in the host galaxy. More generally, what we learn about environments of different SN Types in turn tells us something about the SN progenitor and the stellar populations of galaxies. In particular we think of SNe of Type Ia whose star progenitor systems have not unambiguously been identified yet. A careful investigation of both circumstellar and interstellar environments of these SNe may provide clues on the nature of their progenitors and in some instances allow to test some of the proposed pre-SN scenarios (Boffi \& Branch 1995; Branch et al. 1995). The identification of Type Ia SN star system progenitors is important (among other reasons) because the nature of the progenitors is connected to the use of these $\mathrm{SNe}$ as distance indicators and to derive cosmological parameters (Perlmutter et al. 1998; Riess et al. 1998). In fact to determine the mass and energy density of the Universe from SNe of Type Ia in the Hubble diagram, the evolution with cosmic epoch of both the Type Ia SN rate and their luminosity function are relevant; these functions depend on the nature of the progenitor systems.

In Sect. 2 the sample of galaxies is presented and we explain the selection criteria used. The observations are described and the analysis process is presented in Sect. 3. Our results are summarized in Sect. 4 where we discuss (a) the results from our search for candidate light echoes; (b) some by-products of the present investigation in terms of SN environments and of SN star progenitors. Conclusions and plans for future work are discussed in the last section.

\section{Observations}

\subsection{Sample selection}

Firstly all galaxies have been selected to be parent galaxies of historical supernovae. Many host multiple SN events. Second, echoes, if present, would be resolved with high resolution imaging using the Hubble Space Telescope instrumentation, i.e. their angular size would be $\geq 0.1$ arcsec on the long distance scale (i.e., for a low value of the Hubble's constant). This resulted in a total sample of (North \& South) 172 galaxies, 211 supernovae. Due to time, visibility and weather constraints, of these we observed 38 galaxies, hosting 64 supernovae. Furthermore, for some, their distance is already well determined by means of normal Type Ia supernovae and Cepheid variable stars (e.g. NGC 5253, IC 4182), thus they are are already tied to traditional methods of distance determinations and can be used for comparisons between different methods. Priority was given to such galaxies and to galaxies hosting multiple events. In other regards, our resulting sample is unbiassed and representative of the larger sample.

\subsection{Observing runs}

The data presented in this paper were obtained in three observing runs: one at the $1 \mathrm{~m}$ Jacobus Kapteyn Telescope (JKT) in La Palma, Canary Islands, in October 1994 and two with EFOSC, at the $3.6 \mathrm{~m}$ Cassegrain telescope at the European Southern Observatory, La Silla, Chile, respectively in March and May 1995 (see Tables 1a and 1b). In all runs $B, V$ and $R$ images were taken for each target. Seventeen galaxies were observed with the $1 \mathrm{~m}$ JKT and a further 26 with the $3.6 \mathrm{~m}$ telescope of ESO. All galaxies are listed, in order of increasing Right Ascension, in Tables 1a and $1 \mathrm{~b}$, respectively for the JKT and the ESO runs. Both tables are structured as follows: the galaxy name (NGC, UGC, IC, M) is given in the first column, Right Ascension and declination follow, then the morphological Type of the galaxy is listed in Col. 4. The supernova(e) is (are) given in the fifth column, followed by their offset with respect to the nucleus of the galaxy (unless otherwise noted); finally host galaxy recession velocity, date of observation, exposure times in $B, V$ and $R$, respectively, are given (from Col. 8 through 11). In Table $1 b$ a letter "p" next to the exposure time indicates that polarization measurements were also taken. The polarization results will be presented elsewhere. The $B$ and $V$ bands are in the Johnson system, the $R$ in the Cousins. 
Table 1. a) ${ }^{a}$ All coordinates are from the revised Shapley-Ames catalog, unless otherwise noted (3RC=Third Reference Catalog); ${ }^{b}$ galaxy morphological type from the Asiago Catalog; ${ }^{c}$ offset of the supernova in respect to the nucleus of the galaxy as given in the Asiago Catalog (units: arcsec); ${ }^{d}$ recession velocity from the Asiago Catalog (units: $\mathrm{km} \mathrm{s}^{-1}$ ); ${ }^{e}$ observation date in format year/month/date; ${ }^{f}$ number of exposures taken in each band and exposure times in seconds; ${ }^{g}$ two different fields were taken in order to observe all supernovae; ${ }^{h}$ offset (in arcsec) in respect to a nearby star (Porter 1993). An asterisk next to the galaxy name indicates that no $R$ magnitudes were found in the literature. In these cases to calibrate in $R$ we assumed a $V-R$ appropriate to the morphological type of the galaxy, using the relations given by Buta \& Williams (1995; their Table 6 and Fig. 3)

\begin{tabular}{|c|c|c|c|c|c|c|c|c|c|c|}
\hline Galaxy & R. A. ${ }^{a}$ & Dec. $^{a}$ & Type $^{b}$ & $\mathrm{SN}$ & Offset $^{c}$ & Rec. Vel. ${ }^{d}$ & Date $^{e}$ & $B^{f}$ & $V^{f}$ & $R^{f}$ \\
\hline NGC $23^{*}$ & 000719 & 2538.8 & $\mathrm{SBa}$ & $1955 \mathrm{C}$ & $10 \mathrm{E} 10 \mathrm{~N}$ & 4568 & $94 / 10 / 11$ & $2 \times 900$ & $2 \times 900$ & 900 \\
\hline NGC 210 & 003804 & -1408.8 & $\mathrm{Sb}$ & $1954 \mathrm{R}$ & $86 \mathrm{E} 80 \mathrm{~S}$ & 1768 & $94 / 10 / 05$ & $\begin{array}{l}900 \\
600 \\
230\end{array}$ & $\begin{array}{l}900 \\
790\end{array}$ & 300 \\
\hline $\begin{array}{l}\text { NGC } 224 \\
\text { (M31) }\end{array}$ & 004000 & 4059.7 & $\mathrm{Sb}$ & $1885 \mathrm{~A}$ & $15 \mathrm{~W} 4 \mathrm{~S}$ & -299 & $94 / 10 / 05$ & $\begin{array}{l}3 \times 300 \\
600\end{array}$ & $\begin{array}{l}300 \\
200\end{array}$ & 80 \\
\hline NGC $253^{*}$ & 004508 & -2533.7 & $\mathrm{Sc}$ & $1940 \mathrm{E}$ & $51 \mathrm{~W} 17 \mathrm{~S}$ & 248 & $94 / 10 / 05$ & $2 \times 900$ & $\begin{array}{l}2 \times 900 \\
550\end{array}$ & 900 \\
\hline NGC 488 & $01 \quad 1911$ & 0459.8 & $\mathrm{Sb}$ & $1976 \mathrm{G}$ & $011 \mathrm{~S}$ & 2264 & $94 / 10 / 09$ & $2 \times 900$ & $2 \times 900$ & 900 \\
\hline UGC $2069^{*}$ & $\begin{array}{l}02 \quad 32 \quad 31.9 \\
(3 \mathrm{RC})\end{array}$ & 372527 & $\mathrm{Sd}$ & $1961 \mathrm{P}$ & $036 \mathrm{~S}$ & 3665 & $94 / 10 / 11$ & $2 \times 900$ & $2 \times 900$ & 900 \\
\hline UGC $2105^{*}$ & $\begin{array}{l}023437.7 \\
(3 R C)\end{array}$ & 341257 & $\mathrm{SBa}$ & $1938 \mathrm{~A}$ & $8 \mathrm{~W} 28 \mathrm{~S}$ & 4800 & $94 / 10 / 09$ & $2 \times 1000$ & $2 \times 900$ & 900 \\
\hline NGC 1003 & 023606.2 & 403928 & Scd & $1937 \mathrm{D}$ & $48 \mathrm{E} 1 \mathrm{~S}$ & 585 & $94 / 10 / 05$ & $2 \times 900$ & $2 \times 900$ & 300 \\
\hline NGC 1058 & 024023 & $\begin{array}{ll}37 & 07.8\end{array}$ & $\mathrm{Sc}$ & $\begin{array}{l}1961 \mathrm{~V} \\
1969 \mathrm{~L}\end{array}$ & $\begin{array}{c}76 \mathrm{E} 17 \mathrm{~N} \\
190 \mathrm{E} 110 \mathrm{~S}\end{array}$ & 519 & $94 / 10 / 05$ & $\begin{array}{l}900 \\
600\end{array}$ & $\begin{array}{l}900 \\
300\end{array}$ & 300 \\
\hline NGC $1073 *$ & 024105 & $01 \quad 09.9$ & $\mathrm{SBc}$ & $1962 \mathrm{~L}$ & $10 \mathrm{E} 77 \mathrm{~N}$ & 1874 & $94 / 10 / 05$ & $2 \times 900$ & $2 \times 900$ & 900 \\
\hline UGC $2259^{*}$ & $\begin{array}{l}02 \quad 44 \quad 47.9 \\
(3 R C)\end{array}$ & 371950 & SBdm & $1963 \mathrm{~L}$ & $21 \mathrm{~W} 27 \mathrm{~S}$ & 623 & $94 / 10 / 05$ & $\begin{array}{l}2 \times 900 \\
600\end{array}$ & 900 & 300 \\
\hline NGC $1325^{*}$ & 032213 & -2143.1 & $\mathrm{Sbc}$ & $1975 \mathrm{~S}$ & $53 \mathrm{E} 77 \mathrm{~N}$ & 1670 & $94 / 10 / 11$ & $\begin{array}{l}900 \\
650\end{array}$ & $\begin{array}{l}450 \\
200 \\
400\end{array}$ & 900 \\
\hline NGC 2276 & $07 \quad 1031$ & 8550.9 & $\mathrm{Sc}$ & $\begin{array}{l}1962 \mathrm{Q} \\
1968 \mathrm{~V} \\
1968 \mathrm{~W}\end{array}$ & $\begin{array}{c}34 \mathrm{~W} 11 \mathrm{~S} \\
35 \mathrm{~W} 36 \mathrm{~N} \\
7 \mathrm{~W} 7 \mathrm{~N}\end{array}$ & 2369 & $94 / 10 / 09$ & 1000 & 300 & $(-)$ \\
\hline $\begin{array}{l}\text { NGC } 6946 \\
\text { (field A) }^{g}\end{array}$ & 203348 & 5959.0 & Scd & $\begin{array}{l}1948 \mathrm{~B} \\
1968 \mathrm{D} \\
1980 \mathrm{~K}\end{array}$ & $\begin{array}{c}222 \mathrm{E} 60 \mathrm{~N} \\
45 \mathrm{E} 20 \mathrm{~N} \\
280 \mathrm{E} 165 \mathrm{~S}\end{array}$ & 46 & $94 / 10 / 05$ & $3 \times 900$ & $2 \times 900$ & 900 \\
\hline $\begin{array}{l}\text { NGC } 6946 \\
\text { (field B) }^{g}\end{array}$ & 203348 & 5959.0 & Scd & $\begin{array}{l}1917 \mathrm{~A} \\
1939 \mathrm{C} \\
1969 \mathrm{P}\end{array}$ & $\begin{array}{c}37 \mathrm{~W} 105 \mathrm{~S} \\
215 \mathrm{~W} 24 \mathrm{~N} \\
5 \mathrm{~W} 180 \mathrm{~S}\end{array}$ & 46 & $94 / 10 / 09$ & $2 \times 900$ & $2 \times 900$ & 900 \\
\hline NGC $7177^{*}$ & 215818 & 1729.9 & Sab & $\begin{array}{l}1960 \mathrm{~L} \\
1976 \mathrm{E}\end{array}$ & $\begin{array}{c}2 \mathrm{E} 25 \mathrm{~N}^{h} \\
7 \mathrm{~W} 13 \mathrm{~S}\end{array}$ & 1105 & $94 / 10 / 11$ & $2 \times 900$ & $2 \times 900$ & 900 \\
\hline NGC 7331 & 223447 & 3409.5 & Sbc & 1959D & $32 \mathrm{~W} 13 \mathrm{~N}$ & 794 & $94 / 10 / 09$ & $2 \times 1000$ & $2 \times 900$ & 900 \\
\hline
\end{tabular}

For the JKT observations, only imaging observations were performed. A TeK4 CCD detector was used. This is a high-resolution chip with $1024 \times 1024$ pixels corresponding to a 0.33 arcsec pixel ${ }^{-1}$ image scale. The CCD was used with a gain corresponding to 0.78 electrons per CCD analog-to-digital unit (adu), and the readout noise estimated from the variance of intensity values in the overscan region was measured to be $\sim 4.7$ electrons. Seeing was measured to be $\sim 1$ 1. 5 FWHM typically. Bias (zeroexposures) and flat field frames were taken at the beginning and end of each observing night.

For the ESO observations, both imaging and imaging polarization observations were obtained. We mounted four optical-quality polarizing filters utilizing HN38 Polaroid and anti-reflection coated $\mathrm{MgF}_{2}$ substrates at position angles $0^{\circ} 45^{\circ} 90^{\circ} 135^{\circ}$ in the first filter wheel to be encountered by the light beam after passing through the aperture plate. The ESO CCD No. 26 was used as the detector. This is a high resolution TeK detector with $512 \times 512$ pixels corresponding to a 0.61 arcsec pixel ${ }^{-1}$ image scale. The CCD was used with a gain corresponding to 3.8 electrons per CCD adu, and the readout noise estimated from the variance of intensity values in the overscan region was measured to be $\sim 8.1$ electrons. The CCD saturates at $\sim 30000$ electrons. Seeing was measured to be $\sim 2^{\prime \prime}$ FWHM typically. Bias frames were taken at the beginning and end of each observing night and flat field exposures of the dome interior were also obtained.

\section{Data analysis}

The analysis process primarily fell in three parts: firstly, we performed basic photometric calibration of the data, secondly we made high signal-to-noise images suitable to locate any interesting patches at the site of (or close to) the supernova, thirdly we performed photometric measurements of features visible in the SN environments. 
Table 1. b) ${ }^{a}$ All coordinates are from the revised Shapley-Ames catalog, unless otherwise noted (3RC=Third Reference Catalog); ${ }^{b}$ galaxy morphological type from the Asiago Catalog; ${ }^{c}$ offset of the supernova in respect to the nucleus of the galaxy as given in the Asiago Catalog (units: arcsec); ${ }^{d}$ recession velocity from the Asiago Catalog (units: $\mathrm{km} \mathrm{s}^{-1}$ ); ${ }^{e}$ observation date in format year/month/date; ${ }^{f}$ number of exposures taken in each band and exposure times in seconds; the letter "p" indicates that polarimetry was done; ${ }^{g}$ lost one exposure and then repeated; ${ }^{h}$ offset (in arcsec) in respect to a nearby star (Porter 1993); ${ }^{i}$ astrometric positions of SNe 1923A and 1957D from Pennington et al. (1982); radio positions of SNe 1950B and 1983N from Weiler et al. (1986). An asterisk next to the galaxy name indicates that no $R$ magnitudes were found in the literature. In these cases to calibrate in $R$ we assumed a $V-R$ appropriate to the morphological type of the galaxy, using the relations given by Buta \& Williams (1995; their Table 6 and Fig. 3)

\begin{tabular}{|c|c|c|c|c|c|c|c|c|c|c|}
\hline Galaxy & R. A. ${ }^{a}$ & Dec. $^{a}$ & Type $^{b}$ & $\mathrm{SN}$ & Offset $^{c}$ & Rec. Vel. ${ }^{d}$ & Date $^{e}$ & $B^{f}$ & $V^{f}$ & $R^{f}$ \\
\hline \multirow[t]{2}{*}{ NGC 2935* } & 093427 & -2054.2 & $\mathrm{SBb}$ & $1975 \mathrm{~F}$ & $5 \mathrm{E} 10 \mathrm{~S}$ & 2204 & $95 / 05 / 04$ & & 30 & \\
\hline & & & & & & & & 450 & $4 \times 240 \mathrm{p}$ & 60 \\
\hline \multirow[t]{3}{*}{ NGC 3115} & 100244 & -0728.5 & S0 & 1935B & $36 \mathrm{~W} 60 \mathrm{~N}$ & 698 & $95 / 05 / 04$ & 30 & $\begin{array}{l}4 \times 30 \mathrm{p} \\
5\end{array}$ & 5 \\
\hline & & & & & & & & 240 & $4 \times 240 \mathrm{p}$ & 90 \\
\hline & & & & & & & & 180 & $4 \times 180 \mathrm{p}$ & \\
\hline \multirow[t]{2}{*}{ NGC 3627} & 111738 & 1315.8 & $\mathrm{Sb}$ & $1973 \mathrm{R}$ & $49 \mathrm{~W} 25 \mathrm{~N}$ & 697 & $95 / 05 / 03$ & $2 \times 300$ & $8 \times 300 \mathrm{p}$ & $2 \times 90$ \\
\hline & & & & 1989B & $15 \mathrm{~W} 50 \mathrm{~N}$ & & & & & 10 \\
\hline \multirow[t]{2}{*}{ NGC 4038} & 115919 & -1835.11 & Sm p & $1921 \mathrm{~A}$ & $25 \mathrm{E} 13 \mathrm{~S}$ & 1624 & $95 / 05 / 04$ & 600 & $4 \times 600 \mathrm{p}$ & 180 \\
\hline & & & & $1974 \mathrm{E}$ & $62 \mathrm{E} 42 \mathrm{~S}$ & & & & & \\
\hline \multirow[t]{3}{*}{ NGC $4254^{*}$} & 121617 & 1441.7 & $\mathrm{Sc}$ & $1967 \mathrm{H}$ & $80 \mathrm{E} 19 \mathrm{~S}$ & 2413 & $95 / 05 / 03$ & & 30 & \\
\hline & & & & $1972 Q$ & $15 \mathrm{E} 97 \mathrm{~N}$ & & & 600 & $4 \times 600 \mathrm{p}$ & 180 \\
\hline & & & & $1986 \mathrm{I}$ & $36 \mathrm{E} 16 \mathrm{~S}$ & & & & & \\
\hline \multirow[t]{3}{*}{ NGC 4303} & 121922 & 0445.1 & $\mathrm{Sc}$ & 1926A & $11 \mathrm{~W} 69 \mathrm{~N}$ & 1566 & $95 / 05 / 03$ & & 30 & \\
\hline & & & & $1961 \mathrm{I}$ & $82 \mathrm{E} 12 \mathrm{~S}$ & & & 600 & $4 \times 600 \mathrm{p}$ & 180 \\
\hline & & & & $1964 \mathrm{~F}$ & $28 \mathrm{~W} 1 \mathrm{~S}$ & & & & & \\
\hline \multirow{4}{*}{$\begin{array}{l}\text { NGC } 4321 \\
\text { (M100) }\end{array}$} & 122023.3 & 160600 & $\mathrm{Sc}$ & 1901B & $110 \mathrm{~W} 4 \mathrm{~N} ?$ & 1568 & $95 / 03 / 04$ & 600 & $4 \times 600 \mathrm{p}$ & 180 \\
\hline & & & & 1914A & $24 \mathrm{E} 111 \mathrm{~S}$ & & & & & \\
\hline & & & & $1959 \mathrm{E}$ & $58 \mathrm{E} 21 \mathrm{~S}$ & & & & & \\
\hline & & & & $1979 \mathrm{C}$ & $56 \mathrm{E} 87 \mathrm{~S}$ & & & & & \\
\hline \multirow[t]{2}{*}{ NGC 4382} & 122253 & 1828.0 & S0 p & 1960R & $8 \mathrm{E} 132 \mathrm{~S}$ & 760 & $95 / 05 / 04$ & & 30 & \\
\hline & & & & & & & & $\begin{array}{l}600 \\
300\end{array}$ & $4 \times 600 \mathrm{p}$ & 180 \\
\hline \multirow[t]{3}{*}{ NGC $4424^{*}$} & 122440 & 0941.8 & $\mathrm{Sa} \mathrm{p}$ & $1895 \mathrm{~A}$ & $75 \mathrm{E} 11 \mathrm{~S}$ & 439 & $95 / 05 / 03$ & & 30 & \\
\hline & & & & & & & & 600 & $4 \times 600 \mathrm{p}$ & 180 \\
\hline & & & & & & & $95 / 03 / 05$ & 600 & $4 \times 600 \mathrm{p}$ & 180 \\
\hline NGC $4674^{*}$ & 124327.7 & -082258 & SB0 & $1907 \mathrm{~A}$ & $10 \mathrm{~W} 11 \mathrm{~N}$ & 1301 & $95 / 03 / 05$ & 600 & $2 \times 600 \mathrm{p}$ & 180 \\
\hline \multirow[t]{3}{*}{ NGC 4753} & 124948 & -0055.7 & I0 & $1965 \mathrm{I}$ & $98 \mathrm{~W} 68 \mathrm{~N}$ & 1255 & $95 / 05 / 04$ & & 30 & \\
\hline & & & & $1983 \mathrm{G}$ & $17 \mathrm{~W} 14 \mathrm{~S}$ & & & 600 & $4 \times 600 \mathrm{p}$ & 180 \\
\hline & & & & & & & $95 / 03 / 05$ & 600 & 300 & 90 \\
\hline \multirow{2}{*}{ IC $4237^{*}$} & 13218 & -2052 & $\mathrm{SBb}$ & $1962 \mathrm{H}$ & $19 W 3 \mathrm{~S}$ & 2731 & $95 / 05 / 03$ & & 30 & \\
\hline & & & & & & & & 600 & $4 \times 600 \mathrm{p}$ & 180 \\
\hline \multirow{4}{*}{$\begin{array}{l}\text { NGC } 5236 \\
(\mathrm{M} 83)\end{array}$} & 133410 & -2936.8 & $\mathrm{SBc}$ & 1923A & astrometric $^{i}$ & 506 & $95 / 03 / 04$ & $4 \times 60 \mathrm{p}$ & $8 \times 600 p$ & $4 \times 300 \mathrm{p}$ \\
\hline & & & & 1950B & $\operatorname{radio}^{i}$ & & & & $4 \times 25 \mathrm{p}$ & \\
\hline & & & & 1957D & astrometric ${ }^{i}$ & & $95 / 03 / 05$ & $4 \times 1200$ & & \\
\hline & & & & $\begin{array}{l}1968 \mathrm{~L} \\
1983 \mathrm{~N}\end{array}$ & $\begin{array}{c}5 \mathrm{~W} 0 \\
\text { radio }^{i}\end{array}$ & & & & & \\
\hline \multirow[t]{2}{*}{ NGC 5253} & 133705 & -3123.4 & $\mathrm{I} 0 \mathrm{p}$ & 1895B & $16 \mathrm{E} 23 \mathrm{~N}$ & 403 & $95 / 03 / 04$ & $8 \times 180 \mathrm{p}$ & $4 \times 300 \mathrm{p}$ & $(-)$ \\
\hline & & & & $1972 \mathrm{E}$ & $38 \mathrm{~W} 100 \mathrm{~S}$ & & & & $4 \times 210 \mathrm{p}$ & $(-)$ \\
\hline \multirow{3}{*}{ NGC 5668* } & 143054 & 0440.2 & $\mathrm{Sd}$ & $1952 \mathrm{G}$ & $19 \mathrm{~W} 25 \mathrm{~S}$ & 1584 & $95 / 05 / 03$ & & $\begin{array}{l}8 \times 60 \mathrm{p} \\
30\end{array}$ & \\
\hline & & & & $1954 \mathrm{~B}$ & $2 \mathrm{~W} 20 \mathrm{~S}$ & & & 600 & $4 \times 600 \mathrm{p}$ & 180 \\
\hline & & & & & & & $95 / 03 / 05$ & 600 & 300 & \\
\hline \multirow[t]{2}{*}{ NGC $5857^{*}$} & $\begin{array}{lll}15 & 05 & 11.2\end{array}$ & 194727 & $\mathrm{SBb}$ & $1950 \mathrm{H}$ & $11 \mathrm{E} 22 \mathrm{~S}$ & 4705 & $95 / 05 / 04$ & & 30 & \\
\hline & & & & $1955 \mathrm{M}$ & $27 \mathrm{~W} 25 \mathrm{~N}$ & & & 600 & $4 \times 600 \mathrm{p}$ & 180 \\
\hline NGC $5861^{*}$ & 150633 & 5959.0 & $\mathrm{Sc}$ & 1971D & $33 \mathrm{E} 2 \mathrm{~N}$ & 1851 & $95 / 05 / 04$ & 600 & $4 \times 600 \mathrm{p}$ & 180 \\
\hline NGC 6181* & 163010 & 1955.9 & $\mathrm{Sc}$ & 1926B & $048 \mathrm{~N}$ & 2158 & $95 / 05 / 03$ & & 30 & \\
\hline \multirow[t]{2}{*}{ NGC 6384} & 172959 & $\begin{array}{ll}07 & 05.8\end{array}$ & $\mathrm{Sbc}$ & $1971 \mathrm{~L}$ & $27 \mathrm{E} 20 \mathrm{~N}$ & 1751 & $95 / 05 / 03$ & 600 & $\begin{array}{l}4 \times 600 \mathrm{p} \\
30\end{array}$ & 180 \\
\hline & & & & & & & & 600 & $4 \times 600 \mathrm{p}$ & 180 \\
\hline IC $4719^{*}$ & 182857 & $\begin{array}{llll}-56 & 46 & 12\end{array}$ & I & $1934 \mathrm{~A}$ & $6 \mathrm{E} 13 \mathrm{~S}$ & $(-)$ & $95 / 05 / 04$ & 600 & $5 \times 600^{g} \mathrm{p}$ & 180 \\
\hline IC $4798^{*}$ & $1853 \quad 44.1$ & $\begin{array}{lll}-62 & 11 & 12\end{array}$ & $\mathrm{Sa}$ & 1971R & ? $12 \mathrm{SE}$ & 4182 & $95 / 05 / 04$ & 600 & $4 \times 600 \mathrm{p}$ & 180 \\
\hline NGC 6835* & 195147 & -1242.0 & I0 & $1962 \mathrm{~J}$ & $42 \mathrm{~W} 23 \mathrm{~S}$ & 1557 & $95 / 05 / 03$ & 240 & $4 \times 240 \mathrm{p}$ & 120 \\
\hline \multirow[t]{2}{*}{ NGC $7177^{*}$} & 215818 & 1729.9 & Sab & $1960 \mathrm{~L}$ & $2 \mathrm{E} 25 \mathrm{~N}$ & 1105 & $95 / 05 / 04$ & 300 & $300 \mathrm{p}$ & 120 \\
\hline & & & & $1976 \mathrm{E}$ & $7 \mathrm{~W} 13 \mathrm{~S}$ & & & & & \\
\hline
\end{tabular}




\subsection{Calibration}

First, following standard procedures, the images were debiassed and flat-fielded (using IRAF arithmetic routines). Then spatial registration of the images was performed. For each galaxy, one image was taken as reference. Then the IRAF task IMEXAM was used to measure the pixel coordinates of the peak intensity of an object (or objects) visible in all images (and for all bands), using a centering algorithm. If the nucleus of the galaxy was not suitable, i.e. it was saturated, then the positions of typically three stars were located and taken as reference, otherwise the position of the galaxy nucleus itself was used. All images of each galaxy were then registered with linear shifts and linear interpolation of fluxes (IMSHIFT or IMLINTRAN in IRAF).

After the registration, the sum of the images in the same band was derived to maximize the signal to noise ratio of each color. For each galaxy one $B$ sum, one $V$ sum and one $R$ sum were produced. In addition, we also simply summed all available data in all bands to get a maximum $\mathrm{S} / \mathrm{N}$ intensity image.

The sky levels were estimated with the use of the "median" option of IMSTAT (in IRAF) in an empty window of these images selected by eye. The photometric measurements were made by using PHOT (in noao.digiphot.apphot). PHOT was used for two different operations: to use the CCD image to simulate aperture photometry of the entire galaxy and hence, by comparison to published photometry, derive an absolute calibration of the data; and secondly to obtain aperture photometry of all patches visible around the location of the supernova to investigate the SN environment and locate candidate light echoes. The photometry used for absolute calibration was from the Longo \& de Vaucouleurs (1983) and the de Vaucouleurs \& Longo (1988) aperture photometry catalogs (respectively $B$ and $V$, and $R$ ), not corrected for galactic extinction. The photometric calibration in each band for each individual galaxy was derived by taking the mean value of all apertures in that band. Magnitudes indicated as "uncertain" in the catalogs were not used.

Five galaxies (NGC 3115, 3627, 4303, 4382 and 6384) were saturated in their nuclear regions in the long exposures. Thus the calibration for these was bootstrapped from a single unsaturated short exposure, which was calibrated as above.

No aperture photometry was found in the literature for UGC 2069, UGC 2259, IC 4237, IC 4798 and NGC 4674. The calibration was realized by adopting the total-extinction corrected blue magnitude provided by NED (the NASA Electronic Database) and by adopting standard $B-V$ and $V-R$ colors appropriate to the galaxy morphological types. Aperture magnitudes were derived for aperture values appropriate to each run pixel scale.
The calibration for NGC 1058 and NGC 1073 was done with respect to two calibration stars (one observed in each night of observations). In the case of NGC 1058 this was necessary because literature photometric apertures fell partially outside the region imaged.

The calibration for field A of NGC 6946 was obtained by scaling it to the calibration of field $B$, because the galaxy nucleus does not appear in field $\mathrm{A}$, although the two fields imaged do overlap.

Finally for a number of galaxies, indicated with an asterisk in Tables 1a and 1b, $R$ magnitudes were not available. In these cases we assumed a $V-R$ appropriate to the morphological type of the galaxy, using the relations given by Buta \& Williams (1995; their Table 6 and Fig. 3).

\subsection{Optical structure}

To locate patches of faint light in order to investigate the environments of the supernovae and seek candidate echoes, we firstly simply summed all images in all bands of a given field to make an image with essentially maximum signal to noise $(\mathrm{S} / \mathrm{N})$ : the "total sum" image. We filtered the image with a $3 \times 3$ median to eliminate cosmic rays. To isolate compact, discrete patches of emission from the underlying larger scale complex emission of the host galaxy, we used a digital "unsharp masking" method. The high $\mathrm{S} / \mathrm{N}$ galaxy image was differenced with a $17 \times 17$ median filtered version of the same image. The final output is an image well suited to identify patches of faint light against the background.

In addition to unsharp mask images of the high $\mathrm{S} / \mathrm{N}$ total sum, we also derived in the same way, unsharp mask images of each individual bandpass (in $B, V$ and $R$ ). Figures 1 through 36 show the environments of the individual supernovae as derived from these unsharp masked images: Figs. 1 through 13 are for the JKT observations, the remaining for the ESO. The figures are in order of the galaxy Right Ascension. Each supernova environment is shown in 4 different images: the total sum, followed by the three bands, $B, V$, and $R$. A 30 arcsec side box is shown centered at the SN position and displayed between $-2 \sigma$ and $+3 \sigma$ where $\sigma$ is a robust estimate of the dispersion of intensity levels in the box (specifically half the percentile width corresponding to $\pm 1 \sigma$ for a Gaussian distribution).

\subsection{The supernova environments}

Each of the unsharp masked total sum images was visually inspected and the position of all distinct objects or patches within a 10 arcsec diameter circle centered at the supernova were tabulated. In a few instances the position of patches located outside of the ring was tabulated when these patches appear blue in the images. Keeping these tabulated positions fixed, we then measured their 
brightness in the other three $B, V$ and $R$ images using a fixed aperture of 3 pixel radius for the JKT run and a fixed aperture of 2 pixels for the ESO run (corresponding to 0.99 and 1.22 arcsec respectively).

The limiting magnitude for each box is different due to different photometric conditions, different telescopes, exposure times, and different levels of contamination from the underlying host galaxy. Approximate $B, V$ and $R$ limiting magnitudes are respectively given in the last three columns of Tables $2 \mathrm{a}$ and $2 \mathrm{~b}$. They correspond to a $5 \sigma$ detection, unless otherwise indicated. Each limiting magnitude is calculated over an aperture of 3 and 2 pixels in radius respectively for the JKT and the ESO runs using the $\sigma$ which is the robust estimate of the dispersion of intensity levels in the box (as discussed above). A complete discussion of Tables $2 \mathrm{a}$ and $2 \mathrm{~b}$ is given in Sect. 4 .

Local Galactic extinction corrections were also applied to the resulting photometry, using values taken from the $A_{B}$ of the Third Reference Catalog (RC3; de Vaucouleurs et al. 1986). The corresponding $V$ and $R$ corrections were derived by taking the following relationships from Cardelli et al. (1989): $A_{B}=1.324 \times A_{V}$ and $A_{R}=0.84 \times A_{V}$.

\section{Results}

\subsection{Basic data}

Results from the photometric data reduction are summarized in Tables $2 \mathrm{a}$ and $2 \mathrm{~b}$ (for the JKT and the ESO runs respectively). In the first column the name of the galaxy is given; then the supernova(e) and the SN Type are listed in columns two and three. In the fourth column we list all patches that have been identified at (or close to) the site of the supernova and that were bright enough to be detected during the observation: they are numbered and briefly described by a comment that follows in column five. Such features are close to/within a ring of approximately 5 arcsec in radius centered at the nominal position of the supernova (see Figs. 1 through 36). A major uncertainty in this work is the actual location of the historical supernova. Re--measurement of a small number of plates containing images of the actual event (ongoing work) suggests typical uncertainties of the order of a few arcsec. Hence, for the present purposes, we chose to study a region of 10 arcsec diameter centered at the nominal location of the SN. For each patch the offset coordinates (in arcsec) with respect to the SN position have been calculated and are given in the next two columns. The estimated $B, V$ and $R$ magnitudes and the $B-V$ and $V-R$ colors are listed in Cols. 8 through 12 of these tables. They are all corrected for local Galactic extinction. As mentioned in Sect 3.3, the following three columns give respectively the $B, V$ and $R$ limiting magnitudes corresponding to a $5 \sigma$ detection (but some magnitudes correspond to a $3 \sigma$ detection limit and are indicated with an asterisk in Tables $2 \mathrm{a}$ and $2 \mathrm{~b}$ ). Finally the last three columns give the estimated $1 \sigma$ uncertainties of the measured $B, V$ and $R$ magnitudes. These uncertainties lead to a typical uncertainty of 0.2 magnitude in the colors $B-V$ and $V-R$ (see color/color plots to follow).

The positions of a handful of SNe are accurately known from radio observations or astrometric measurements. Pennington et al. (1982) provide accurate astrometric positions of SNe 1923A and 1957D (in NGC 5236). For other two supernovae in the same galaxy, SNe 1950B and $1983 \mathrm{~N}$, accurate positions are derived from radio observations (Weiler et al. 1986). Given these absolute positions, the supernovae were located with respect to the SN 1957D position, which was identified by looking at the SN field observed by Long et al. (1992). The position of SN 1960L is accurately known from astrometry (Porter 1993). In the case of SN 1989B we have collected a wide variety of positional information. The position of SN 1961V in NGC 1058 well agrees with the radio position given in Cowan et al. (1988). Finally the position of SN 1885A in NGC 224 (M31) is in very good agreement with the coordinates of its supernova remnant as measured from Hubble Space Telescope Wide Field Planetary Camera-2 images by Fesen et al. (1998). Hence, in these cases, we are much more confident of the location of the original event in our images.

\subsection{Description of supernova environments:}

In the following we describe the SN environments presented in Figs. 1 through 36. All sites are carefully analyzed and plausible candidates indicated. The 5 arcsec radius ring centered at the nominal position of the supernova is depicted at each site. Each figure can be considered as a finding chart corresponding to each supernova. We also include some comparison to the same environments as observed with the Wide Field Planetary Camera-2 (WFPC2) in archival Hubble Space Telescope (HST) proposals when available.

\section{JKT Run:}

NGC 23 - SN 1955C: patch 2, south-west of the SN, is red in $B-V$ and is within imaging limits from the ground. It is probably just part of the body of the galaxy.

NGC 210 - SN 1954R: one patch is clearly visible at the northern edge of the ring; it is very blue in both colors. Classified as a candidate (see Table 3 ).

NGC 224 - SN 1885A: five small patches $(\leq 1$ arcsec) have been detected. They all are red and bright (brighter than 20th $V$ magnitude). The galaxy light might contaminate the environment.

NGC 253 - SN 1940E: two bright patches; in both cases the $B-V$ is very red and the $V-R$ very blue. Patch 1 appears compact and isolated; patch 2 is extended and arc-like. More structure is visible outside of the 5 arcsec ring and is possibly just part of the arm of the galaxy.

NGC 488 - SN 1976G: one very red and bright extended patch (far off North to the assumed SN position). 
Table 2. a) ${ }^{a}$ The SN Type was taken from Branch 1990 and/or from the Sternberg Catalog; ":" after a Type indicates that according to the Sternberg Catalog there is uncertainty; both Types are classified spectroscopically, but the Type II are also classified according to the shape of the light curve $(\mathrm{P}=$ plateau; $\mathrm{L}=$ linear $)$ as given by the Sternberg Catalog. This means that whenever a Type II is indicated as a Type IIP or IIL the Sternberg classification was used; for some objects only the Sternberg classification Type is found and is indicated in parenthesis; for SNe 1940E and 1963L the two sources disagree: both are given; ${ }^{b}$ the offsets are in respect to the location of the supernova and are in units of arcsec; ${ }^{c}$ "indef" indicates that the IRAF program "phot" did not calculate the magnitude in some band and thus the color could not be derived; the (-) indicates no images were taken in that band. ${ }^{d}$ limiting magnitudes corresponding to a $5 \sigma$ detection limit; an asterisk next to the magnitude indicates that the magnitude corresponds to a $3 \sigma$ detection limit: see text for details; ${ }^{e}$ standard deviations relative to the observed $B$, $V$ and $R$ magnitudes

\begin{tabular}{|c|c|c|c|c|c|c|c|c|c|c|c|c|c|c|c|c|c|}
\hline Galaxy & $\mathrm{SN}$ & SN Type ${ }^{a}$ & patch & Class & $\Delta x^{b}$ & $\Delta y^{b}$ & $B^{c}$ & $V^{c}$ & $R^{c}$ & $B-V^{c}$ & $V-R^{c}$ & $B_{\lim }^{d}$ & $V_{\lim }^{d}$ & $R_{\lim }^{d}$ & $\sigma_{B}{ }^{e}$ & $\sigma_{V}^{e}$ & $\sigma_{R}^{e}$ \\
\hline NGC 23 & $1955 \mathrm{C}$ & $(-)$ & 2 & compact/gal.? & $5.55 \mathrm{~W}$ & $1.04 \mathrm{~S}$ & 22.42 & 21.79 & 21.47 & 0.63 & 0.32 & 23.41 & 22.84 & 22.36 & 0.09 & 0.08 & 0.10 \\
\hline NGC 210 & $1954 \mathrm{R}$ & $(-)$ & 4 & compact & $0.48 \mathrm{~W}$ & $5.75 \mathrm{~N}$ & 21.56 & 21.85 & 22.12 & -0.30 & -0.27 & 23.73 & 23.82 & 23.46 & 0.04 & 0.05 & 0.08 \\
\hline NGC 224 & $1885 \mathrm{~A}$ & I & 1 & compact & $1.14 \mathrm{E}$ & $1.65 \mathrm{~N}$ & 20.25 & 19.07 & 18.75 & 1.17 & 0.32 & 20.92 & 19.85 & 19.32 & 0.12 & 0.11 & 0.13 \\
\hline \multirow[t]{4}{*}{ (M31) } & & & 2 & compact & $3.13 \mathrm{E}$ & $2.68 \mathrm{~S}$ & 20.84 & 19.82 & 19.08 & 1.02 & 0.75 & 20.92 & 19.85 & 19.32 & 0.20 & 0.21 & 0.18 \\
\hline & & & 3 & patchy & $2.09 \mathrm{E}$ & $2.78 \mathrm{~S}$ & 20.86 & 19.54 & 18.83 & 1.31 & 0.71 & 20.92 & 19.85 & 19.32 & 0.21 & 0.16 & 0.14 \\
\hline & & & 4 & compact & $2.17 \mathrm{~W}$ & $4.02 \mathrm{~S}$ & 20.52 & 19.74 & 19.13 & 0.78 & 0.61 & 20.92 & 19.85 & 19.32 & 0.15 & 0.20 & 0.18 \\
\hline & & & 5 & patchy & $2.16 \mathrm{~W}$ & $4.01 \mathrm{~S}$ & 20.51 & 19.73 & 19.12 & 0.78 & 0.61 & 20.92 & 19.85 & 19.32 & 0.15 & 0.19 & 0.18 \\
\hline \multirow[t]{2}{*}{ NGC 253} & $1940 \mathrm{E}$ & $(-) /(\mathrm{I})$ & 1 & compact & $1.75 \mathrm{E}$ & $0.23 \mathrm{~N}$ & 20.69 & 19.77 & 19.86 & 0.92 & -0.09 & 21.93 & 21.18 & 21.15 & 0.07 & 0.06 & 0.07 \\
\hline & & & 2 & arc-like & $1.12 \mathrm{E}$ & $1.43 \mathrm{~S}$ & 21.51 & 20.46 & 20.63 & 1.05 & -0.17 & 21.93 & 21.18 & 21.15 & 0.15 & 0.11 & 0.13 \\
\hline NGC 488 & $1976 \mathrm{G}$ & $(-)$ & 1 & tail/gal.? & $0.33 \mathrm{~W}$ & $6.31 \mathrm{~N}$ & 22.14 & 20.82 & 19.71 & 1.33 & 1.11 & 23.41 & 22.48 & 21.57 & 0.07 & 0.05 & 0.04 \\
\hline UGC 2069 & $1961 \mathrm{P}$ & Ia & & nothing & & & & & & & & & & & & & \\
\hline \multirow[t]{2}{*}{ UGC 2105} & $1938 \mathrm{~A}$ & $(-)$ & 2 & point-like & $0.34 \mathrm{E}$ & $3.61 \mathrm{~S}$ & 24.13 & 24.4 & 23.77 & -0.26 & 0.63 & $25.19^{*}$ & $24.82 *$ & $24.25^{*}$ & 0.15 & 0.25 & 0.24 \\
\hline & & & 3 & compact & $11.74 \mathrm{E}$ & $12.4 \mathrm{~S}$ & 22.06 & indef & 25.53 & indef & indef & $25.19^{*}$ & $24.82^{*}$ & $24.25^{*}$ & 0.03 & $(-)$ & $(-)$ \\
\hline \multirow[t]{2}{*}{ NGC 1003} & 1937D & Ia & 2 & patchy & $4.32 \mathrm{~W}$ & $4.02 \mathrm{~S}$ & 22.45 & 22.34 & 22.12 & 0.12 & 0.21 & 24.70 & 24.44 & 23.87 & 0.03 & 0.04 & 0.05 \\
\hline & & & 3 & patchy & $3.17 \mathrm{~W}$ & 0.00 & 24.53 & 23.31 & 23.04 & 1.22 & 0.27 & 24.70 & 24.44 & 23.87 & 0.19 & 0.08 & 0.11 \\
\hline \multirow[t]{3}{*}{ NGC 1058} & $1961 \mathrm{~V}$ & $\mathrm{~V}$ & 1 & $\operatorname{arc}$ & $0.86 \mathrm{~W}$ & $1.65 \mathrm{~S}$ & 22.55 & 23.66 & 22.84 & -1.11 & 0.82 & 24.67 & 24.35 & 23.05 & 0.05 & 0.13 & 0.19 \\
\hline & & & 2 & $\operatorname{arc}$ & $0.87 \mathrm{~W}$ & $1.54 \mathrm{~S}$ & 22.57 & 23.51 & 22.86 & -0.93 & 0.64 & 24.67 & 24.35 & 23.05 & 0.05 & 0.11 & 0.20 \\
\hline & 1969L & IIP & & nothing & & & & & & & & & & & & & \\
\hline NGC 1073 & $1962 \mathrm{~L}$ & $\mathrm{Ib}$ & 3 & extended & $2.43 \mathrm{E}$ & $9.62 \mathrm{~S}$ & 23.15 & 22.83 & 22.3 & 0.32 & 0.54 & 24.81 & 24.62 & 23.97 & 0.06 & 0.05 & 0.06 \\
\hline \multirow[t]{2}{*}{ UGC 2259} & $1963 \mathrm{~L}$ & $(-) /(\mathrm{I})$ & 1 & compact & $5.56 \mathrm{E}$ & $1.22 \mathrm{~S}$ & 22.03 & 21.94 & 22.07 & 0.09 & -0.13 & 24.28 & 24.01 & 22.78 & 0.03 & 0.04 & 0.11 \\
\hline & & & 3 & compact & $6.04 \mathrm{~W}$ & $2.83 \mathrm{~N}$ & 23.59 & 23.61 & 22.61 & -0.02 & 0.99 & 24.28 & 24.01 & 22.78 & 0.10 & 0.16 & 0.17 \\
\hline NGC 1325 & $1975 \mathrm{~S}$ & (II:) & & nothing & & & & & & & & & & & & & \\
\hline \multirow[t]{6}{*}{ NGC 2276} & 1962Q & $(-)$ & 1 & compact & $1.25 \mathrm{E}$ & $7.16 \mathrm{~S}$ & 19.21 & 18.80 & $(-)$ & 0.41 & $(-)$ & 23.40 & 21.63 & $(-)$ & 0.01 & 0.03 & $(-)$ \\
\hline & $1968 \mathrm{~V}$ & (II) & 1 & compact & $4.34 \mathrm{E}$ & $4.22 \mathrm{~N}$ & 20.57 & 20.18 & $(-)$ & 0.39 & $(-)$ & 23.31 & 21.63 & $(-)$ & 0.03 & 0.08 & $(-)$ \\
\hline & & & 2 & compact & $4.96 \mathrm{E}$ & $1.08 \mathrm{~N}$ & 20.14 & 19.63 & $(-)$ & 0.51 & $(-)$ & 23.31 & 21.63 & $(-)$ & 0.02 & 0.05 & $(-)$ \\
\hline & & & 3 & compact & $1.76 \mathrm{E}$ & $4.53 \mathrm{~S}$ & 20.20 & 19.88 & $(-)$ & 0.32 & $(-)$ & 23.31 & 21.63 & $(-)$ & 0.02 & 0.06 & $(-)$ \\
\hline & & & 4 & compact & $3.67 \mathrm{~W}$ & $0.85 \mathrm{~S}$ & 21.62 & 21.51 & $(-)$ & 0.12 & $(-)$ & 23.31 & 21.63 & $(-)$ & 0.06 & 0.22 & $(-)$ \\
\hline & 1968W & $(-)$ & 1 & patchy & $2.66 \mathrm{~W}$ & $0.59 \mathrm{~N}$ & 21.71 & 22.06 & $(-)$ & -0.35 & $(-)$ & $23.37^{*}$ & $22.18^{*}$ & $(-)$ & 0.09 & 0.35 & $(-)$ \\
\hline \multirow[t]{15}{*}{ NGC 6946} & $1917 \mathrm{~A}$ & (II) & 1 & patchy & $3.94 \mathrm{E}$ & $1.77 \mathrm{~N}$ & 21.37 & 21.01 & 20.86 & 0.36 & 0.14 & 21.97 & 22.09 & 21.85 & 0.13 & 0.08 & 0.09 \\
\hline & & & 2 & patchy & $2.48 \mathrm{E}$ & $1.65 \mathrm{~S}$ & 21.24 & 21.31 & 21.49 & -0.07 & -0.17 & 21.97 & 22.09 & 21.85 & 0.12 & 0.11 & 0.16 \\
\hline & & & 3 & compact & $2.78 \mathrm{E}$ & $4.77 \mathrm{~S}$ & 20.77 & 20.65 & 20.54 & 0.11 & 0.12 & 21.97 & 22.09 & 21.85 & 0.08 & 0.06 & 0.07 \\
\hline & & & 4 & patchy & $0.89 \mathrm{~W}$ & $3.88 \mathrm{~N}$ & 19.79 & 19.20 & 18.78 & 0.59 & 0.42 & 21.97 & 22.09 & 21.85 & 0.03 & 0.02 & 0.02 \\
\hline & & & 5 & compact & $0.73 \mathrm{~W}$ & $4.56 \mathrm{~N}$ & 19.42 & 18.86 & 18.47 & 0.57 & 0.39 & 21.97 & 22.09 & 21.85 & 0.03 & 0.01 & 0.01 \\
\hline & $1939 \mathrm{C}$ & (I:) & 1 & compact & $0.06 \mathrm{E}$ & $2.34 \mathrm{~S}$ & 20.26 & 19.61 & 19.20 & 0.65 & 0.40 & 22.77 & 22.84 & 22.47 & 0.03 & 0.02 & 0.02 \\
\hline & & & 2 & compact & $2.09 \mathrm{~W}$ & $2.42 \mathrm{~N}$ & 22.42 & 22.05 & 21.69 & 0.37 & 0.35 & 22.77 & 22.84 & 22.47 & 0.17 & 0.11 & 0.11 \\
\hline & & & 3 & patchy & $2.10 \mathrm{~W}$ & $2.45 \mathrm{~N}$ & 22.41 & 22.05 & 21.71 & 0.36 & 0.35 & 22.77 & 22.84 & 22.47 & 0.17 & 0.11 & 0.11 \\
\hline & $1948 B$ & IIP & 1 & compact & $0.53 \mathrm{E}$ & $1.91 \mathrm{~S}$ & 20.32 & 20.42 & 20.09 & -0.10 & 0.32 & 21.85 & 22.04 & 21.65 & 0.06 & 0.05 & 0.05 \\
\hline & & & 2 & extended & $3.03 \mathrm{~W}$ & $4.40 \mathrm{~N}$ & 18.41 & 18.14 & 17.63 & 0.26 & 0.52 & 21.85 & 22.04 & 21.65 & 0.02 & 0.01 & 0.01 \\
\hline & $1968 \mathrm{D}$ & (II) & 1 & compact & $1.03 \mathrm{E}$ & $3.47 \mathrm{~N}$ & 21.66 & 21.45 & 21.47 & 0.21 & -0.02 & 22.22 & 22.19 & 21.88 & 0.14 & 0.12 & 0.15 \\
\hline & $1969 \mathrm{P}$ & $(-)$ & 1 & extended & $0.25 \mathrm{~W}$ & $4.50 \mathrm{~N}$ & 17.43 & 17.00 & 16.70 & 0.44 & 0.30 & 22.68 & 22.90 & 22.47 & 0.01 & 0.01 & 0.01 \\
\hline & & & 2 & compact & $0.28 \mathrm{~W}$ & $3.62 \mathrm{~N}$ & 18.11 & 17.61 & 17.22 & 0.50 & 0.39 & 22.68 & 22.90 & 22.47 & 0.01 & 0.01 & 0.01 \\
\hline & $1980 \mathrm{~K}$ & IIL & 1 & compact & $2.67 \mathrm{E}$ & $2.12 \mathrm{~S}$ & 22.12 & 21.85 & 21.74 & 0.27 & 0.11 & 22.77 & 23.13 & 22.85 & 0.42 & 0.15 & 0.16 \\
\hline & & & 2 & compact & $5.12 \mathrm{E}$ & $0.003 \mathrm{~N}$ & 21.87 & 22.46 & 22.23 & -0.59 & 0.24 & 22.77 & 23.13 & 22.85 & 0.14 & 0.08 & 0.09 \\
\hline \multirow[t]{2}{*}{ NGC 7177} & $1960 \mathrm{~L}$ & $(-)$ & 1 & compact & $0.51 \mathrm{E}$ & $0.59 \mathrm{~N}$ & 23.68 & 23.19 & 23.48 & 0.50 & -0.30 & 24.34 & 24.46 & 23.69 & 0.13 & 0.08 & 0.19 \\
\hline & $1976 \mathrm{E}$ & $(-)$ & 1 & compact/gal.? & $1.31 \mathrm{E}$ & $0.02 \mathrm{~S}$ & 17.08 & 16.21 & 15.78 & 0.88 & 0.42 & 22.62 & 22.20 & 21.85 & 0.01 & 0.01 & 0.01 \\
\hline \multirow[t]{3}{*}{ NGC 7331} & 1959D & IIL & 1 & patchy & $1.53 \mathrm{E}$ & $1.69 \mathrm{~N}$ & 22.95 & 22.90 & 22.68 & 0.05 & 0.22 & $23.84^{*}$ & $23.17^{*}$ & $22.75^{*}$ & 0.16 & 0.28 & 0.34 \\
\hline & & & 2 & compact & $3.16 \mathrm{E}$ & $1.82 \mathrm{~S}$ & 20.61 & 20.41 & 20.16 & 0.21 & 0.25 & 23.29 & 22.61 & 22.20 & 0.02 & 0.03 & 0.04 \\
\hline & & & 3 & patchy & $0.93 W$ & $3.30 \mathrm{~N}$ & 22.29 & 21.78 & 21.49 & 0.50 & 0.29 & 23.29 & 22.61 & 22.20 & 0.09 & 0.10 & 0.12 \\
\hline
\end{tabular}


Table 2. b) ${ }^{a}$ The SN Type was taken from Branch 1992 and/or from the Sternberg Catalog; “"” after a Type indicates that according to the Sternberg Catalog there is uncertainty; both Types are classified spectroscopically, but the Type II are also classified according to the shape of the light curve $(\mathrm{P}=$ plateau; $\mathrm{L}=$ linear $)$ as given by the Sternberg Catalog. This means that whenever a Type II is indicated as a Type IIP or IIL the Sternberg classification was used; for some objects only the Sternberg classification Type is found and is indicated in parenthesis; for SNEe 1961I, 1964F and 1967H the two sources disagree: both are given; ${ }^{b}$ the offsets are in respect to the location of the supernova and are in units of arcsec. ${ }^{c}$ "indef" indicates that the IRAF program "phot" did not calculate the magnitude in some band and thus the color could not be derived; the (-) indicates no images were taken in that band; "sat." for SN 1968L in NGC 5236 indicates that the R band was saturated near the nucleus.

${ }^{d}$ limiting magnitudes corresponding to a $5 \sigma$ detection limit; an asterisk next to the magnitude indicates that the magnitude corresponds to a $3 \sigma$ detection limit: see text for details; ${ }^{e}$ standard deviations relative to the observed $B, V$ and $R$ magnitudes

\begin{tabular}{|c|c|c|c|c|c|c|c|c|c|c|c|c|c|c|c|c|c|}
\hline Galaxy & $\mathrm{SN}$ & SN Type ${ }^{a}$ & patch & Class & $\Delta x^{b}$ & $\Delta y^{b}$ & $B^{c}$ & $V^{c}$ & $R^{c}$ & $B-V^{c}$ & $V-R^{c}$ & $B_{\mathrm{lim}}^{d}$ & $V_{\lim }^{d}$ & $R_{\mathrm{lim}}^{d}$ & $\sigma_{B}^{e}$ & $\sigma_{V}^{e}$ & $\sigma_{R}^{e}$ \\
\hline NGC 2935 & $1975 \mathrm{~F}$ & $(\mathrm{Pec})$ & 1 & compact & $0.84 \mathrm{E}$ & $1.11 \mathrm{~N}$ & 16.58 & 15.56 & 15.25 & 1.03 & 0.31 & 21.18 & 20.25 & 19.92 & 0.01 & 0.01 & 0.01 \\
\hline NGC 3115 & 1935B & $(-)$ & 1 & compact & $4.37 \mathrm{E}$ & $5.83 \mathrm{~S}$ & 22.64 & 21.84 & 20.79 & 0.80 & 1.05 & 24.86 & 25.10 & 23.41 & 0.03 & 0.01 & 0.02 \\
\hline \multirow[t]{2}{*}{ NGC 3627} & 1973R & IIP & 1 & tail & $0.30 \mathrm{E}$ & $6.14 \mathrm{~S}$ & 20.81 & 20.59 & 20.34 & 0.22 & 0.24 & 21.19 & 21.10 & 20.95 & 0.15 & 0.14 & 0.12 \\
\hline & 1989B & $\mathrm{Ia}$ & 1 & compact & $0.20 \mathrm{~W}$ & $1.58 \mathrm{~S}$ & 19.90 & 19.93 & 19.91 & -0.02 & 0.02 & $20.27^{*}$ & $20.26^{*}$ & $20.15^{*}$ & 0.26 & 0.26 & 0.29 \\
\hline \multirow[t]{2}{*}{ NGC 4038} & 1921A & $(-)$ & & nothing & & & & & & & & & & & & & \\
\hline & $1974 \mathrm{E}$ & $(-)$ & & nothing & & & & & & & & & & & & & \\
\hline \multirow[t]{6}{*}{ NGC 4254} & $1967 \mathrm{H}$ & $? /(\mathrm{II})$ & 1 & tail & $0.33 \mathrm{~W}$ & $6.42 \mathrm{~N}$ & 19.06 & 18.84 & 18.42 & 0.22 & 0.42 & 22.58 & 22.27 & 21.94 & 0.01 & 0.01 & 0.01 \\
\hline & & & 2 & compact & $0.94 \mathrm{E}$ & $1.91 \mathrm{~N} ?$ & 22.33 & 22.02 & 21.84 & 0.31 & 0.18 & 22.58 & 22.27 & 21.94 & 0.17 & 0.17 & 0.20 \\
\hline & & & 3 & compact & $3.47 \mathrm{E}$ & $6.17 \mathrm{~S}$ & 21.22 & 20.80 & 20.12 & 0.42 & 0.68 & 22.58 & 22.27 & 21.94 & 0.06 & 0.06 & 0.04 \\
\hline & $1972 \mathrm{Q}$ & (IIP) & 1 & patchy & $5.04 \mathrm{~W}$ & $1.17 \mathrm{~N}$ & 21.95 & 21.62 & 21.32 & 0.32 & 0.30 & 22.60 & 22.41 & 22.19 & 0.12 & 0.10 & 0.10 \\
\hline & & & 2 & patchy & $1.01 \mathrm{~W}$ & $2.05 \mathrm{~N}$ & 21.91 & 21.77 & 21.59 & 0.14 & 0.18 & 22.60 & 22.41 & 22.19 & 0.11 & 0.12 & 0.13 \\
\hline & 1986I & II & 1 & pathcy & $1.33 \mathrm{E}$ & $1.60 \mathrm{~N}$ & 19.76 & 19.68 & 19.55 & 0.09 & 0.12 & 20.84 & 20.58 & 20.38 & 0.08 & 0.09 & 0.10 \\
\hline \multirow[t]{3}{*}{ NGC 4303} & 1926A & II & 1 & patchy & $1.06 \mathrm{E}$ & $0.83 \mathrm{~N}$ & 21.96 & 21.96 & 21.79 & -0.01 & 0.17 & $22.15^{*}$ & $22.15^{*}$ & $21.89^{*}$ & 0.30 & 0.30 & 0.33 \\
\hline & $1961 \mathrm{I}$ & II/(II:) & 1 & compact & $0.48 \mathrm{E}$ & $1.24 \mathrm{~N}$ & 19.85 & 20.04 & 19.93 & -0.19 & 0.11 & 22.72 & 22.64 & 22.29 & 0.02 & 0.02 & 0.03 \\
\hline & $1964 \mathrm{~F}$ & II/(I) & 1 & compact & $1.81 \mathrm{E}$ & $0.52 \mathrm{~S}$ & 21.16 & 21.33 & 21.33 & -0.17 & 0.01 & 21.88 & 21.88 & 21.69 & 0.11 & 0.13 & 0.15 \\
\hline NGC 4321 & 1901B & (I) & 1 & patchy & $1.98 \mathrm{E}$ & $0.90 \mathrm{~S}$ & 22.47 & 22.22 & 21.94 & 0.25 & 0.28 & 22.76 & 22.45 & 22.09 & 0.17 & 0.18 & 0.19 \\
\hline \multirow[t]{6}{*}{ (M100) } & & & 2 & patchy & $7.34 \mathrm{~W}$ & $2.07 \mathrm{~S}$ & 21.61 & 21.32 & 20.91 & 0.30 & 0.40 & 22.76 & 22.45 & 22.09 & 0.08 & 0.08 & 0.07 \\
\hline & & & 3 & patchy & $1.36 \mathrm{~W}$ & $6.02 \mathrm{~N}$ & 20.79 & 20.38 & 19.40 & 0.41 & 0.97 & 22.76 & 22.45 & 22.09 & 0.04 & 0.03 & 0.02 \\
\hline & $1914 \mathrm{~A}$ & $(-)$ & 2 & compact & $5.44 \mathrm{~W}$ & $1.20 \mathrm{~N}$ & 23.40 & 23.27 & 22.84 & 0.14 & 0.43 & 24.62 & 24.29 & 23.67 & 0.07 & 0.09 & 0.10 \\
\hline & $1959 \mathrm{E}$ & (I) & 1 & patchy & $0.55 \mathrm{E}$ & $6.39 \mathrm{~S}$ & 20.99 & 20.87 & 20.08 & 0.12 & 0.79 & 21.75 & 21.36 & 20.94 & 0.11 & 0.14 & 0.10 \\
\hline & & & 2 & patchy & $1.59 \mathrm{~W}$ & $3.55 \mathrm{~N}$ & 21.13 & 20.77 & 20.44 & 0.37 & 0.32 & 21.75 & 21.36 & 20.94 & 0.12 & 0.12 & 0.14 \\
\hline & $1979 \mathrm{C}$ & IIL & 1 & compact & $3.97 \mathrm{E}$ & $0.60 \mathrm{~S}$ & 21.04 & 20.73 & 20.32 & 0.31 & 0.41 & 22.58 & 22.35 & 22.01 & 0.05 & 0.05 & 0.05 \\
\hline \multirow[t]{2}{*}{ NGC 4382} & 1960R & I & 1 & compact & $1.23 \mathrm{~W}$ & $2.57 \mathrm{~N}$ & 24.71 & 24.30 & 23.68 & 0.41 & 0.62 & $25.65^{*}$ & $25.54^{*}$ & $24.16^{*}$ & 0.16 & 0.12 & 0.24 \\
\hline & & & 2 & patchy & $0.72 \mathrm{~W}$ & $5.25 \mathrm{~S}$ & indef & 24.35 & 22.80 & indef & 1.55 & 25.09 & 24.98 & 23.60 & & 0.12 & 0.11 \\
\hline $\begin{array}{l}\text { NGC } 4424 \\
\text { (May) }\end{array}$ & 1895A & I & 1 & compact & $2.59 \mathrm{E}$ & $6.20 \mathrm{~S}$ & 24.37 & 22.83 & 21.62 & 1.54 & 1.21 & 25.60 & 25.05 & 24.31 & 0.08 & 0.03 & 0.02 \\
\hline $\begin{array}{l}\text { NGC } 4424 \\
\text { (March) }\end{array}$ & 1895A & $\mathrm{I}$ & 1 & compact & $2.84 \mathrm{E}$ & $6.01 \mathrm{~S}$ & 24.19 & 23.10 & 21.85 & 1.09 & 1.25 & 25.31 & 24.62 & 22.89 & 0.08 & 0.06 & 0.09 \\
\hline \multirow[t]{2}{*}{ NGC 4674} & $1907 \mathrm{~A}$ & (I) & 1 & tail/gal. & $1.70 \mathrm{~W}$ & $2.30 \mathrm{~S}$ & 21.83 & 21.13 & 20.56 & 0.70 & 0.57 & 23.50 & 22.37 & 21.92 & 0.05 & 0.07 & 0.06 \\
\hline & & & 2 & compact & $1.73 \mathrm{~W}$ & $2.17 \mathrm{~S}$ & 21.87 & 21.13 & 20.56 & 0.74 & 0.57 & 23.50 & 22.37 & 21.92 & 0.05 & 0.07 & 0.06 \\
\hline NGC 4753 & $1965 \mathrm{I}$ & Ia & 2 & compact & $6.22 \mathrm{~W}$ & $0.95 \mathrm{~S}$ & 23.89 & 22.69 & 22.31 & 1.20 & 0.38 & 25.46 & 25.28 & 24.39 & 0.06 & 0.02 & 0.04 \\
\hline & $1983 \mathrm{G}$ & Ia & & nothing & & & & & & & & & & & & & \\
\hline NGC 4753 & $1965 \mathrm{I}$ & Ia & & nothing & & & & & & & & & & & & & \\
\hline (March) & $1983 \mathrm{G}$ & Ia & & nothing & & & & & & & & & & & & & \\
\hline IC 4237 & $1962 \mathrm{H}$ & (II) & 1 & tail & $3.85 \mathrm{E}$ & $6.12 \mathrm{~S}$ & 20.77 & 20.67 & 20.43 & 0.10 & 0.24 & 21.74 & 21.65 & 21.33 & 0.07 & 0.09 & 0.10 \\
\hline & & & 2 & compact & $2.34 \mathrm{~W}$ & $1.58 \mathrm{~N}$ & 20.55 & 20.52 & 20.27 & 0.03 & 0.25 & 21.74 & 21.65 & 21.33 & 0.06 & 0.08 & 0.08 \\
\hline NGC 5236 & 1923A & (II) & 1 & compact & $0.43 \mathrm{E}$ & $0.14 \mathrm{~N}$ & 19.16 & 18.90 & 18.45 & 0.26 & 0.46 & 20.70 & 20.41 & 19.88 & 0.05 & 0.05 & 0.06 \\
\hline (M83) & & & 2 & compact & $3.76 \mathrm{~W}$ & $1.82 \mathrm{~N}$ & 19.10 & 18.63 & 17.97 & 0.47 & 0.65 & 20.70 & 20.41 & 19.88 & 0.05 & 0.04 & 0.04 \\
\hline & 1950B & $(-)$ & 1 & compact & $4.04 \mathrm{~W}$ & $0.18 \mathrm{~S}$ & 20.72 & 20.67 & 20.13 & 0.05 & 0.55 & 21.45 & 21.00 & 20.32 & 0.11 & 0.16 & 0.18 \\
\hline & 1957D & $(-)$ & 2 & compact & $6.00 \mathrm{~W}$ & $1.90 \mathrm{~N}$ & 20.91 & 20.77 & 19.81 & 0.14 & 0.97 & 21.28 & 21.24 & 20.82 & 0.16 & 0.14 & 0.09 \\
\hline & $1968 \mathrm{~L}$ & IIP & 1 & compact & $5.25 \mathrm{~W}$ & $2.87 \mathrm{~N}$ & 20.24 & 20.67 & sat. & -0.43 & $(-)$ & $20.98^{*}$ & $20.28^{*}$ & sat. & $(-)$ & $(-)$ & $(-)$ \\
\hline & $1983 \mathrm{~N}$ & $\mathrm{Ib}$ & 1 & double & $0.06 \mathrm{E}$ & $0.95 \mathrm{~N}$ & 21.70 & 21.34 & 20.94 & 0.35 & 0.40 & 21.94 & 21.64 & 21.13 & 0.17 & 0.17 & 0.18 \\
\hline NGC 5253 & 1895B & (I) & 1 & patchy & $6.38 \mathrm{E}$ & $0.42 \mathrm{~S}$ & 21.75 & 21.76 & $(-)$ & -0.01 & $(-)$ & 22.52 & 22.23 & $(-)$ & 0.11 & 0.14 & $(-)$ \\
\hline & & & 2 & compact & $2.75 \mathrm{~W}$ & $1.30 \mathrm{~S}$ & 21.19 & 20.93 & $(-)$ & 0.25 & $(-)$ & 22.52 & 22.23 & $(-)$ & 0.06 & 0.06 & $(-)$ \\
\hline & $1972 \mathrm{E}$ & Ia & 1 & compact & $1.44 \mathrm{E}$ & $1.66 \mathrm{~S}$ & indef & 24.37 & $(-)$ & indef & $(-)$ & 24.94 & 24.40 & $(-)$ & $(-)$ & 0.21 & $(-)$ \\
\hline NGC 5668 & $1952 \mathrm{G}$ & $(-)$ & 1 & compact & $0.08 \mathrm{~W}$ & $1.56 \mathrm{~N}$ & 21.59 & 21.23 & 21.00 & 0.37 & 0.23 & 23.12 & 22.78 & 22.52 & 0.05 & 0.05 & 0.05 \\
\hline & & & 2 & compact & $0.56 \mathrm{E}$ & $2.91 \mathrm{~S}$ & 23.48 & 22.98 & 22.84 & 0.50 & 0.14 & $23.67^{*}$ & $23.33^{*}$ & $23.07^{*}$ & 0.30 & 0.26 & 0.29 \\
\hline & 1954B & I & 1 & patchy & $3.01 \mathrm{E}$ & $8.00 \mathrm{~N}$ & 21.79 & 21.51 & 21.09 & 0.28 & 0.42 & 22.36 & 21.97 & 21.68 & 0.13 & 0.14 & 0.13 \\
\hline & & & 2 & compact? & $2.62 \mathrm{E}$ & $6.95 \mathrm{~N}$ & 22.63 & 22.26 & 21.83 & 0.37 & 0.44 & $22.91^{*}$ & $22.52^{*}$ & $22.23^{*}$ & 0.28 & 0.28 & 0.25 \\
\hline NGC 5668 & $1952 \mathrm{G}$ & $(-)$ & 1 & compact & $0.31 \mathrm{~W}$ & $1.34 \mathrm{~N}$ & 22.61 & 21.10 & $(-)$ & 1.51 & $(-)$ & 23.07 & 22.04 & $(-)$ & 0.14 & 0.10 & $(-)$ \\
\hline (March) & & & 2 & patchy & $0.26 \mathrm{~W}$ & $1.44 \mathrm{~N}$ & 22.61 & 21.13 & $(-)$ & 1.48 & $(-)$ & 23.07 & 22.04 & $(-)$ & 0.14 & 0.10 & $(-)$ \\
\hline & & & 3 & patchy & $0.30 \mathrm{~W}$ & $1.37 \mathrm{~N}$ & 22.60 & 21.11 & $(-)$ & 1.50 & $(-)$ & 23.07 & 22.04 & $(-)$ & 0.14 & 0.10 & $(-)$ \\
\hline & 1954B & I & & & & & & & & & & & & & & & \\
\hline NGC 5857 & $1950 \mathrm{H}$ & $(-)$ & 1 & compact & $1.36 \mathrm{E}$ & $3.01 \mathrm{~N}$ & 22.07 & 21.66 & 21.46 & 0.41 & 0.20 & 23.88 & 23.33 & 22.81 & 0.04 & 0.05 & 0.06 \\
\hline & $1955 \mathrm{M}$ & $(-)$ & & & & & & & & & & & & & & & \\
\hline NGC 5861 & 1971D & $(-)$ & 1 & compact & $5.07 \mathrm{~W}$ & $0.63 \mathrm{~S}$ & 23.79 & 23.45 & 23.72 & 0.34 & -0.27 & 24.82 & 24.72 & 24.05 & 0.09 & 0.07 & 0.16 \\
\hline NGC 6181 & 1926B & $(-)$ & 1 & double & $2.98 \mathrm{E}$ & $4.19 \mathrm{~S}$ & 21.06 & 20.95 & 20.90 & 0.11 & 0.05 & 23.00 & 22.55 & 22.22 & 0.04 & 0.05 & 0.06 \\
\hline & & & 2 & system & $1.04 \mathrm{~W}$ & $2.29 \mathrm{~N}$ & 20.79 & 20.51 & 20.21 & 0.28 & 0.30 & 23.00 & 22.55 & 22.22 & 0.03 & 0.03 & 0.03 \\
\hline NGC 6384 & $1971 \mathrm{~L}$ & Ia & 1 & patchy & $0.36 \mathrm{~W}$ & $2.25 \mathrm{~N}$ & 22.65 & 22.44 & 22.04 & 0.21 & 0.39 & $23.30^{*}$ & $22.86^{*}$ & $22.80^{*}$ & 0.20 & 0.24 & 0.23 \\
\hline IC 4719 & 1934A & $(-)$ & 1 & compact & $1.51 \mathrm{E}$ & $4.21 \mathrm{~S}$ & 21.38 & 21.27 & 21.08 & 0.11 & 0.19 & 22.09 & 21.69 & 21.40 & 0.11 & 0.15 & 0.16 \\
\hline & & & 2 & patchy & $5.02 \mathrm{~W}$ & $3.82 \mathrm{~S}$ & 22.36 & 22.10 & 21.65 & 0.26 & 0.45 & $22.64^{*}$ & $22.25^{*}$ & $21.96^{*}$ & 0.28 & 0.31 & 0.27 \\
\hline IC 4798 & $1971 \mathrm{R}$ & $(-)$ & 1 & tail/gal.? & $6.72 \mathrm{~W}$ & $7.18 \mathrm{~N}$ & 18.32 & 17.41 & 17.59 & 0.91 & -0.18 & 21.38 & 20.76 & 20.17 & 0.01 & 0.01 & 0.02 \\
\hline & & & 2 & compact & $2.97 \mathrm{~W}$ & $9.29 \mathrm{~S}$ & 19.68 & 19.15 & 18.79 & 0.53 & 0.36 & 21.38 & 20.76 & 20.17 & 0.04 & 0.05 & 0.06 \\
\hline NGC 6835 & 1962J & Ia & 1 & compact & $4.38 \mathrm{~W}$ & $3.39 \mathrm{~N}$ & 23.10 & 22.64 & 21.16 & 0.46 & 1.48 & $23.56^{*}$ & $22.92^{*}$ & $22.40^{*}$ & 0.24 & 0.28 & 0.29 \\
\hline NGC 7177 & 1960L & $(-)$ & 1 & compact & $5.63 \mathrm{E}$ & $5.17 \mathrm{~N}$ & 22.64 & 23.09 & 22.31 & -0.45 & 0.78 & 24.08 & 24.56 & 23.00 & 0.06 & 0.06 & 0.12 \\
\hline & & & 2 & compact & $0.63 \mathrm{E}$ & $1.06 \mathrm{~N}$ & 23.29 & 23.50 & 23.53 & -0.21 & -0.02 & $24.64^{*}$ & $25.11^{*}$ & $23.56^{*}$ & 0.11 & 0.08 & 0.35 \\
\hline & $1976 \mathrm{E}$ & $(-)$ & 1 & compact/gal.? & $1.58 \mathrm{E}$ & $0.007 \mathrm{~S}$ & 17.41 & 16.40 & 16.08 & 1.01 & 0.32 & 21.00 & 20.11 & 19.79 & 0.01 & 0.01 & 0.01 \\
\hline
\end{tabular}


It looks like a small tail "departing" from the galaxy nucleus.

UGC 2069 - SN 1961P: no patches are distinguishable within the region just around the $\mathrm{SN}$; an isolated compact object (possibly a star?) is to the south-east of the SN nominal position. An arc of compact bright objects lies well to the northern edge of the SN sections.

UGC 2105 - SN 1938A: two patches are located, one to the south-west of the supernova (within the 5 arcsec ring) and the other well further out in the same direction. The latter looks very blue from visual inspection. The photometry indicates that the first patch is blue in color, and that the second one is very blue and faint in visual magnitude. They are both brighter than $3 \sigma$ detection limits.

NGC 1003 - SN 1937D: two patches are identified in the immediate surroundings of the SN location: they are both displaced in the $x$ coordinate from the SN position and are compact. The hosting galaxy was oberved with WFPC-2 on the HST. The region around the SN is not very crowded and compact sources of emission are visible. Two groups of stars are observed South of the SN immediate environment and appear unrelated to the region of interest.

NGC 1058 - SNe 1961V, 1969L: an elongated patch is clearly visible at the location of SN $1961 \mathrm{~V}$; in $V$ it appears broken up into different smaller patches: a couple of these are faint and extremely blue in $B-V$. The region of SN 1969L is empty. SN $1961 \mathrm{~V}$ classified as a candidate (see Table 3).

NGC 1073 - SN 1962L: no patches are visible visible in the immediate surroundings of the SN. An extended patch instead is visible off the 5 arcsec ring to the south-east of the SN position.

UGC 2259 - SN 1963L: a blue and compact object is visible to the south-east of the SN position on the edge of the ring. Two patches slightly off the ring, to the northwest, appear blue and patchy and should be further investigated. Classified as a candidate (see Table 3).

NGC 1325 - SN 1975S: no patches are visible either within or outside the ring.

NGC 2276 - SNe 1962Q, 1968V, 1968W: no $R$ band observations are available. The first two SNe lie in crowded regions. South-east of SN 1962Q a bright and compact patch of light is seen. At least four relatively bright patches are identified at around the location of SN 1968V. Further observations are needed (in the $R$ band for example). At the SN $1968 \mathrm{~W}$ position some blue emission brighter than a $3 \sigma$ magnitude is visible. The last supernova is close to the nucleus of the galaxy.

NGC 6946/field A+field B - SNe 1917A, 1939C, 1948B, 1968D, 1969P, 1980K: at the sites of SNe 1948B, $1968 \mathrm{D}$ and $1980 \mathrm{~K}$ some patches are located: they all are bright and quite blue. SN $1980 \mathrm{~K}$ is likely the most interesting of all $\mathrm{SNe}$ in this galaxy; in fact from all the data collected at radio wavelengths over a long period of time (Weiler et al. 1986, 1991) and from the fact that its optical counterpart has also been identified, it appears a good candidate to look at because of dense circumstellar and interstellar environments. At the sites of SNe 1917A and 1969P bright blue patches are observed. Star-like objects appear in the environment. In the case of SN 1939C some objects are visible as well. SNe 1917A, 1969P and 1980K are classified as candidates (see Table 3 ).

NGC 7177 - SNe 1960L, 1976E: at the location of SN 1960L some patches are seen. A blue one is seen at the location of the supernova, brighter than a $5 \sigma$ detection. As already discussed the position of SN 1960L is accurately known from Porter (1993) and thus the identification of a blue patch at the location of the supernova is of great interest and calls for further investigation. At the site of SN $1976 \mathrm{E}$ a very bright and red compact object is observed. SN 1960L is classified as a candidate (see Table 3).

NGC 7331 - SN 1959D: patch 1 is within a $3 \sigma$ detection limit; patch 2 is brighter and blue, although appears compact on the image and might be only a star. Patch 3 is also blue and compact. These patches are also visible on the archival WFPC-2 image that we analyzed. With WFPC-2 it is clear that there are groups of stars present (possibly new forming stars) and/or HII regions belonging to a galaxy spiral arm.

\section{ESO Runs:}

NGC 2935 - SN 1975F: one extremely bright compact object not too far off the SN position; red in $B-V$. It looks similar to what is seen at the location of SN $1976 \mathrm{E}$ in NGC 7177.

NGC 3115 - SN 1935B: one red and compact object is visible to the south-east of the SN location and at the edge of the ring. Several other patches of the same kind are found all around the region.

NGC 3627 - SNe 1973R, 1989B: in the case of SN 1973R a bright blue elongated object is visible. Almost at the location of SN 1989B a roundish fuzzy and very blue (in both $B-V$ and $V-R$ ) patch of light is present. The emission we see is within a $3 \sigma$ detection limit. Both SN environments are part of spiral arms and very crowded. On the basis of our selection criteria, both supernovae are classified as candidates (see Table 3).

NGC 4038 - SNe 1921A, 1974E: no patches are visible within 5 arcsec of the nominal positions of these two supernovae.

NGC 4254 - SNe 1967H, 1972Q, 1986I: several bright and blue features are seen at the locations of these supernovae. The SN sites are quite crowded though and the visible features might simply belong to the spiral arms of the galaxy.

NGC 4303 - SNe 1926A, 1961I, 1964F: at all these sites some features are present. From visual inspection and its blue color, the faint arc-shaped patch at the SN 1926A site looks promising. It is brighter than a $3 \sigma$ detection limit. The extended, bright, round patch at the site of SN 1961I is quite likely to be a HII region or young open cluster. 
SN 1926A is classified as a candidate (see Table 3). Both SNe $1926 \mathrm{~A}$ and $1964 \mathrm{~F}$ are visible in the WFPC-2 images of the host galaxy. We believe we can confirm what we noticed in our ground based observations.

NGC 4321 (M100) - SNe 1901B, 1914A, 1959E, 1979C: all environments show relatively bright and blue patches of light. The SN 1979C environment is possibly the most promising, as it is known from radio observations to be characterized by a dense environment (Weiler et al. 1981; Weiler et al. 1986). This SN has also been optically identified. Only in this one case (in this galaxy) is an emission patch clearly visible within 5 arcsec from the SN nominal position. Less compelling evidence has been gathered for the other supernovae. SN 1979C is classified as a candidate (see Table 3 ). The positions of SNe $1959 \mathrm{E}$ and $1979 \mathrm{C}$ are also identified on some archival WFPC-2 observations. Multiple sources are present in the immediate surroundings of the SNe. Many may well be star clusters, others more directly related to the SN event. For example we know the optical counterpart to SN 1979C has been identified among all other sources (Van Dyk, private communication).

NGC 4382 - SN 1960R: two very faint patches are detected within the immediate $\mathrm{SN}$ regions. All images appear to be very noisy. Patch 1 is within a $3 \sigma$ detection limit.

NGC 4424 - SN 1895A: from both runs (March and May 1995) one faint and compact patch is visible to the south-east of the SN location (at the edge of the 5 arcsec ring); it is very red in $B-V$.

NGC 4674 - SN 1907A: an elongated structure lying to the south-west of the SN is visible. Some other patch is also visible far off the SN nominal position. Both are red in color and possibly are just part of the main body of the galaxy.

NGC 4753 - SN 1965I, 1983G: at both locations some structure is found. The March 1995 run is very noisy and does not provide any information. Only at the location of SN 1965I (May run) is some faint structure visible to the south-west of the supernova.

IC 4237 - SN 1962H: a bright, blue, roundish patch is visible in all sections and is located (within 5 arcsec from the SN position) at the end of an arc-like structure possibly part of a spiral arm of the galaxy. Classified as a candidate (see Table 3 ).

NGC5236 (M83) - SNe 1923A, 1950B, 1957D, 1968L, 1983N: all patches at all locations appear to be very bright and blue (both in the $B-V$ and in the $V-R$ colors). $A t$ the site of SN $1983 \mathrm{~N}$ two compact patches are visible. Note that the positions of these supernovae are known accurately (see Sect. 4.1). In all these cases the SN star progenitors are likely to have been massive (two SNe are confirmed Type II's and one is a Type Ib; SN 1957D is likely to be a Type II, as possibly inferred from the radio data, see Weiler et al. 1986). Around such progenitors dense circumstellar and possibly interstellar environ- ments are expected, thus allowing for the formation of light echoes. The best bets may be SNe 1957D and 1983N. SNe 1957D and 1983N are classified as candidates (see Table 3).

NGC 5253 - SN 1895B, 1972E: no $R$ band observations are available. Some structure is present close to the location of SN 1895B. Some patches are also visible near the location of SN 1972E. SN 1895B is also on the edge of some WFPC-2 images. As single images in different filters were found the image quality of the summed image is not optimal. We can see some structure in the surroundings of the supernova but this HST imaging does not contribute additional information on the presence of reflected light.

NGC 5668 - SNe 1952G, 1954B: both show some faint structures slightly north of the SN positions. The surrounding environments are crowded. Both supernovae lie not too far from the nucleus of the galaxy and belong to spiral arms. The May observations are slightly affected by saturation; the March ones have a poorer signal-to-noise ratio (the galaxy was observed well into twilight). For the March run no $R$ band observations are available. WFPC-2 observations resolve the underlying spiral arm structure. The environments are not particularly crowded and at the location of SN 1952G the same single bright object observed from the ground is also observed with HST, i.e. it remains unresolved with WFPC-2.

NGC 5857 - SN 1950H, 1955M: a compact and blue patch is found at the location of SN 1950H. This SN lies close to the nuclear part of the galaxy.

NGC 5861 - SN 1971D: a patch is visible within the 5 arcsec ring, very close to the location of the SN. Classified as a candidate (see Table 3). WFPC-2 observations show emission close to the SN position. Yet the HST images resolve the spiral arm and thus many compact objects are indeed visible, making it difficult to discriminate easily among them.

NGC 6181 - SN 1926B: a blue and bright doublelobed object is visible at the site of this supernova. It lies within the 5 arcsec ring and occupies more than half of it. Classified as a candidate (see Table 3). This SN lies on the top left corner of the $\mathrm{PC}$ images retrieved from the archive. The SN environment is not very crowded, at the extreme end of the spiral arm. Some faint compact sources of emission are visible.

NGC 6384 - SN 1971L: a blue arc-shaped patch is present just close to the center of the section and is brighter than the $3 \sigma$ detection limit. HST images resolve the underlying spiral structure and several compact sources of emission are present within the uncertainty ring. Some structure is seen around the nominal position of the SN.

IC 4719 - SN 1934A: along the circumference of the 5 arcsec ring a series of faint and blue knots are visible; some are within a $5 \sigma$ detection and some within a $3 \sigma$. 


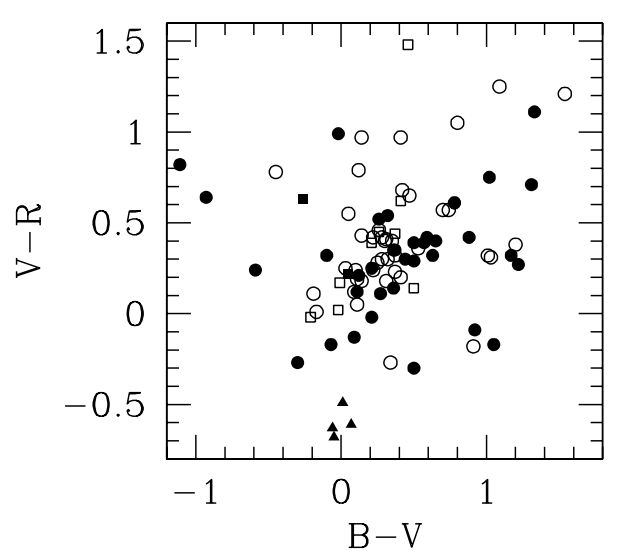

Fig. 37. Color/color plot for all patches at/around the location of the historical supernovae for both observing runs. In this figure only patches whose $B, V$ and $R$ magnitudes are brighter than the calculated limiting magnitudes (corresponding either to a $5 \sigma$ or a $3 \sigma$ detection, as presented in Sect. 4.1) are plotted. The ESO observations are the open circles $(5 \sigma ; 40$ objects) and the open squares $(3 \sigma ; 11$ objects $)$; the JKT are the filled circles $(5 \sigma ; 37$ objects) and the filled squares $(3 \sigma ; 2$ objects); SN $1991 \mathrm{~T}$ is represented by the filled triangles (data from Schmidt et al. 1994)

IC 4798 - SN 1971R: two bright patches are visible, one, patch 1 , possibly belongs to the nuclear part of the galaxy. The second one is to the south-west of the SN.

NGC 6835 - SN 1962J: a patch is visible within the ring, to the east of the supernova. It is within a $3 \sigma$ detection limit.

NGC 7177 - SNe 1960L, 1976E: at the location of SN 1960L some patches are seen as in the JKT observations. A blue one is seen at the location of the supernova and is within a $3 \sigma$ detection limit. SN $1960 \mathrm{~L}$ is classified as a candidate (see Table 3 ).

\subsection{Candidate light echoes}

On the basis of what we see at various SN positions, we classify some optical emission patches as likely candidate light echoes and present them in Table 3 . These candidates are close to the SN nominal position (typically within the 5 arcsec ring, but some lie as far as 6 arcsec away), appear blue in color and are compact. A bonus is granted when the SN position is accurately known (see Sect. 4.1). All data in Table 3 are derived from Tables $1 \mathrm{a}$ through $2 \mathrm{~b}$.

\subsection{Correlations and $S N$ environments}

In order to investigate the SN environments we have made color/color plots for the features visible at the SN environment. In Fig. 37 we plot the two color diagram of all patches whose $B, V$ and $R$ magnitudes are brighter than the calculated limiting magnitudes (corresponding

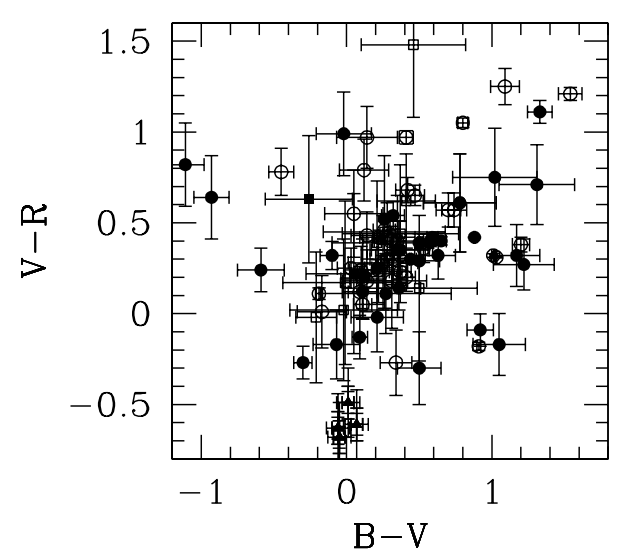

Fig. 38. The same as Fig. 37 but the corresponding $B-V$ and $V-R$ error bars are plotted. The corresponding standard deviations in the three band of observations are given in Tables $2 \mathrm{a}$ and $2 \mathrm{~b}$

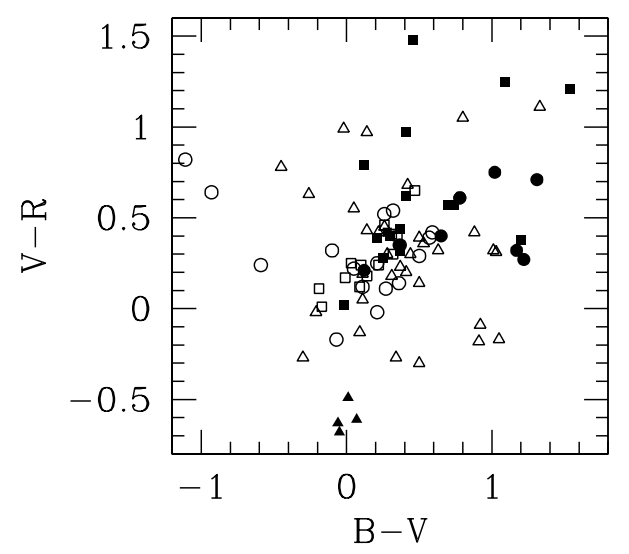

Fig. 39. Figure 39 is the same as Fig. 37 but allowing for a differentiation of the SN Types: all filled symbols refer to Type I SNe (Ia's), all open ones to Type II's (the only Type Ib SN fulfilling the selection criteria for this plot is SN 1983N and is plotted among the Type II as they likely derive from similarly massive star progenitors; in our samples there are no Type Ic SNe). The ESO observations are the squares; the JKT the circles. The empty triangles refer to peculiar supernovae and those whose Type is unknown according to the classification in Tables 2a and 2b. The filled triangles are SN 1991T (data from Schmidt et al. 1994)

either to a $5 \sigma$ or a $3 \sigma$ detection as presented in Sects. 3.3 and 4.1$)$. The ESO observations are the open circles $(5 \sigma$; 40 objects) and the open squares $(3 \sigma ; 11$ objects); the JKT are the filled circles $(5 \sigma ; 37$ objects) and the filled squares $(3 \sigma ; 2$ objects); SN $1991 \mathrm{~T}$ (data from Schmidt et al. 1994) is represented by the filled triangles. All patches appear distributed within the following intervals: $-1.2<(B-V)<1.8$ and $-0.8<(V-R)<1.6$, centered at around 0.3 in both colors. Typically both colors are affected by a 0.2 magnitude error as can be seen in Fig. 38, where for each data point the error bars estimated from the $1 \sigma$ uncertainties in Tables $2 \mathrm{a}$ and $2 \mathrm{~b}$ are plotted. 
Table 3. All data in this table were derived from Tables 1a through $2 \mathrm{~b}$

\begin{tabular}{lccccccc}
\hline \hline \multirow{2}{*}{ Galaxy } & SN & SN Type & patch & $\Delta x$ & $\Delta y$ & $B-V$ & $V-R$ \\
\hline \multirow{2}{*}{ NGC 210 } & $1954 \mathrm{R}$ & $(-)$ & 4 & $0.48 \mathrm{~W}$ & $5.75 \mathrm{~N}$ & -0.30 & -0.27 \\
NGC 1058 & $1961 \mathrm{~V}$ & $\mathrm{~V}$ & 1 & $0.86 \mathrm{~W}$ & $1.65 \mathrm{~S}$ & -1.11 & 0.82 \\
& & & 2 & $0.87 \mathrm{~S}$ & $1.54 \mathrm{~S}$ & -0.93 & 0.64 \\
UGC 2259 & $1963 \mathrm{~L}$ & $(-) /(\mathrm{I})$ & 1 & $5.56 \mathrm{E}$ & $1.22 \mathrm{~S}$ & 0.09 & -0.13 \\
& & & 3 & $6.04 \mathrm{~W}$ & $2.83 \mathrm{~N}$ & -0.02 & 0.99 \\
NGC 3627 & $1973 \mathrm{R}$ & IIP & 1 & $0.30 \mathrm{E}$ & $6.14 \mathrm{~S}$ & 0.22 & 0.24 \\
& $1989 \mathrm{~B}$ & $\mathrm{Ia}$ & 1 & $0.20 \mathrm{~W}$ & $1.58 \mathrm{~S}$ & -0.02 & 0.02 \\
NGC 4303 & $1926 \mathrm{~A}$ & II & 1 & $1.06 \mathrm{E}$ & $0.83 \mathrm{~N}$ & -0.01 & 0.17 \\
NGC 4321 & $1979 \mathrm{C}$ & IIL & 1 & $3.97 \mathrm{E}$ & $0.60 \mathrm{~S}$ & 0.31 & 0.41 \\
IC 4237 & $1962 \mathrm{H}$ & (II) & 2 & $2.34 \mathrm{~W}$ & $1.58 \mathrm{~N}$ & 0.03 & 0.25 \\
NGC 5236 & $1957 \mathrm{D}$ & $(-)$ & 2 & $6.00 \mathrm{~W}$ & $1.90 \mathrm{~N}$ & 0.14 & 0.97 \\
& $1983 \mathrm{~N}$ & Ib & 1 & $0.06 \mathrm{E}$ & $0.95 \mathrm{~N}$ & 0.35 & 0.40 \\
NGC 5861 & $1971 \mathrm{D}$ & $(-)$ & 1 & $5.07 \mathrm{~W}$ & $0.63 \mathrm{~S}$ & 0.34 & -0.27 \\
NGC 6181 & $1926 \mathrm{~B}$ & $(-)$ & 1 & $2.98 \mathrm{E}$ & $4.19 \mathrm{~S}$ & 0.11 & 0.05 \\
& & & 2 & $1.04 \mathrm{~W}$ & $2.29 \mathrm{~N}$ & 0.28 & 0.30 \\
NGC 6946 & $1917 \mathrm{~A}$ & (II) & 1 & $3.94 \mathrm{E}$ & $1.77 \mathrm{~N}$ & 0.36 & 0.14 \\
& & & 2 & $2.48 \mathrm{E}$ & $1.65 \mathrm{~S}$ & -0.07 & 0.17 \\
& & & 3 & $2.78 \mathrm{E}$ & $4.77 \mathrm{~S}$ & 0.11 & 0.12 \\
& & & 4 & $0.89 \mathrm{~W}$ & $3.88 \mathrm{~N}$ & 0.59 & 0.42 \\
& & & 5 & $0.73 \mathrm{~W}$ & $4.56 \mathrm{~N}$ & 0.57 & 0.39 \\
& $1969 \mathrm{P}$ & $(-)$ & 1 & $0.25 \mathrm{~W}$ & $4.50 \mathrm{~N}$ & 0.44 & 0.30 \\
& & & 2 & $0.28 \mathrm{~W}$ & $3.62 \mathrm{~N}$ & 0.50 & 0.39 \\
& $1980 \mathrm{~K}$ & IIL & 1 & $2.67 \mathrm{E}$ & $2.12 \mathrm{~S}$ & 0.27 & 0.11 \\
& & & 2 & $5.12 \mathrm{E}$ & $0.003 \mathrm{~N}$ & -0.59 & 0.24 \\
NGC 7177 & $1960 \mathrm{~L}$ & & & & & & \\
& $($ JKT) & $(-)$ & 1 & $5.63 \mathrm{E}$ & $5.17 \mathrm{~N}$ & -0.45 & 0.78 \\
& & & 2 & $0.63 \mathrm{E}$ & $1.06 \mathrm{~N}$ & -0.21 & -0.02 \\
& & & 1 & $0.51 \mathrm{E}$ & $0.59 \mathrm{~N}$ & 0.50 & -0.30 \\
\hline
\end{tabular}

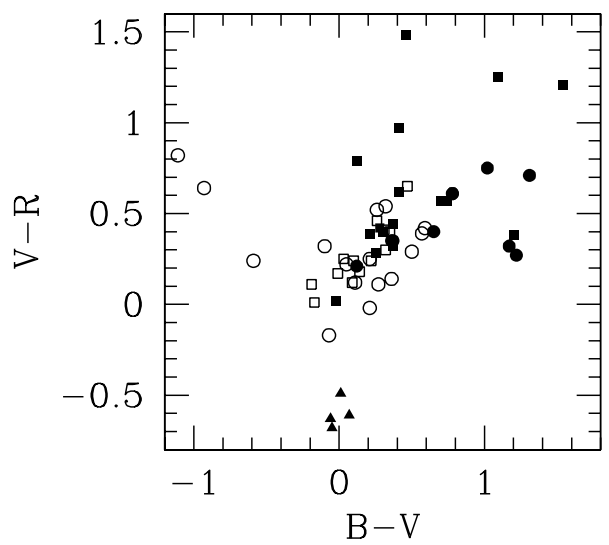

Fig. 40. The same as Fig. 39 except that unknown/peculiar SNe are not plotted

Figure 39 is the same as Fig. 37 but allowing for a differentiation of the SN Types: all filled symbols refer to Type I SNe (Ia's), all open ones to Type II's (Type Ib's are inserted in this group as they likely derive from similarly massive star progenitors; in our samples there are no Type Ic SNe). The ESO observations are the squares; the JKT, the circles. The empty triangles refer to peculiar supernovae and those whose Type is unknown according to the classification in Tables $2 \mathrm{a}$ and $2 \mathrm{~b}$. The filled triangles are SN 1991T (from Schmidt et al. 1994). We have always followed the spectroscopic classification of Branch (1990) and therefore SNe 1961I, 1961V and 1964F have been considered Type II, while SNe 1940E, 1960L, 1963L

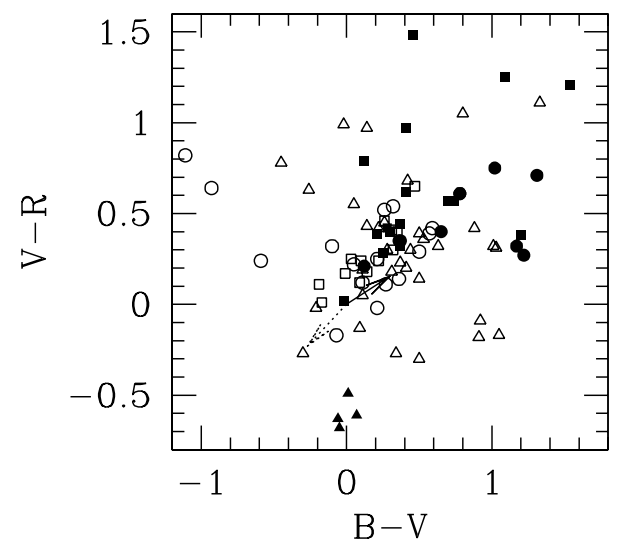

Fig. 41. The same as Fig. 39. The reddening vector (solid line), derived from the standard reddening law of Rieke \& Lebofski (1985), and the scattering vector are also plotted. See text for details

and $1967 \mathrm{H}$ unknown. SN 1961V (in NGC 1058) which was classified as a Type V by Zwicky (1965) might not be a pure SN event (Goodrich et al. 1989; Filippenko et al. 1995) and yet can be as useful as any other object if it produced a light echo. It is clear that different Types of SNe are located in different regions of the color/color plot: the emission patches associated to Type Is are mostly located at $B-V$ and $V-R$ redder than $\sim 0.10$, while Type IIs are on average bluer than this value in both colors. Unknown/peculiars are randomly distributed; the location of the peculiar Type Ia SN 1991T is somewhat 


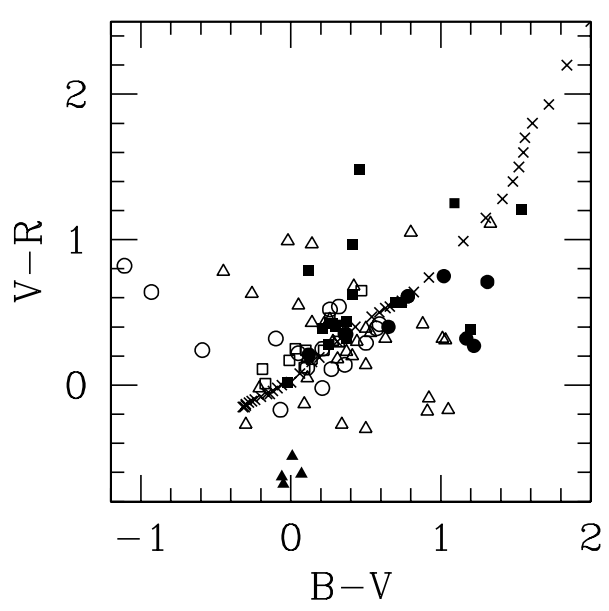

Fig. 42. The same as Fig. 39. Main Sequence star colors are also plotted for comparison. See text for details

consistent with the other peculiars. This is what might be expected from the distribution of different Type SNe in different galaxies and in different parts of the parent galaxies themselves. SNe II/Ib likely derive from massive progenitors in spiral galaxies and thus are associated to star-formation regions where a strong $\mathrm{H} \alpha$ emission is present (Van Dyk et al. 1996 and references therein). This would tend to cause a high positive value of $(V-R)$. Type Ia $\mathrm{SNe}$, instead, possibly derive from a wider variety of stellar environments (as they are observed both in elliptical and spiral galaxies; see Barth et al. 1996 for references).

In Fig. 40 we plot all supernovae except the unknown/peculiar. We note that known Type SNe cover a much smaller portion of the color/color plane than unknown/peculiar.

In Fig. 41 we plot for comparison both a reddening vector (solid arrow) and a scattering vector (dotted arrow) to ascertain in what proportions these two physical components affect the colors of our sample of patches. The reddening vector was derived by assuming the Rieke \& Lebofski (1985) reddening law: $E(B-V) / E(V-R)=$ 0.78. The effect of scattering, also, was estimated using scattering cross-sections as tabulated in Sparks (1994). With these numbers and assuming wavelengths of 4400 , 5500,6400 for the $B V R$ filters, light which has been scattered is always bluer by -0.3 in $B-V$ and -0.2 in $V-R$ than the unscattered illuminating source. For illustration, the scattering vector shown originates at $B-V=V-R=$ 0 , which is a reasonable estimate of the colors of a supernova at peak luminosity in many cases (D. Branch, private communication). In fact, we cannot be sure in detail what the colors of the time integrated supernova light curves are since decay rates can be quite wavelength dependent, and reddening may also play a role. Nevertheless, outliers, and especially blue outliers, in these diagrams should be considered as objects worthy of additional follow-up observations as potential echoes, and yet strict SN 1991T look-alikes, i.e. definite echoes (Sparks et al. 1999), are not present in the sample.

Finally, for reference, in Fig. 42 colors of Main Sequence stars of all spectral types were plotted over our sample (these colors were taken from Zombek 1992). The distribution of patches is broadly consistent with the locations of these Main Sequence stars. This distribution covers also a similar region to a composite stellar population as a function of age as was found by plotting an evolutionary track kindly provided by C. Leitherer. We therefore infer that for the most part we are simply seeing stars or star clusters. However there are in addition patches with both unusually red and unusually blue colors which should be considered with special care.

\section{Conclusions and future work}

We have looked for candidate light echoes around 64 supernovae of all Types in 38 galaxies. From this groundbased photometric data we notice optical emission at the SN nominal positions. The color distribution of these patches is broad, and generally consistent with stellar population colors, possibly with some reddening. However there are in addition patches with both unusually red and unusually blue colors. We expect light echoes to be blue, and while none of the objects are quite as blue in $V-R$ as the known light echo of SN1991T, there are features that are unusually blue and we identify these as candidate echoes for follow-on space and ground observations.

We also made reference to archival WFPC-2/HST observations of fourteen SN fields. These showed that some of the patches resolve into multiple point sources, while others do not. The absence of multi-color information within the HST data precludes us from narrowing down our candidate list further, since at WFC resolution we do not expect the SN remnants to show significant spatial extent. As discussed in the text and in Sparks (1994) only polarimetric observations of a field many arcsec in extent around the SN position can succeed in determining whether (or not) there is an extended structure. This is the basis for future observations using the Advanced Camera on the Hubble Space Telescope.

From the color/color plots we notice that indeed different SN Types are characterized by different environments. SNe Ia tend to be redder than $(B-V)=0-0.1$ and $(V-R)=0-0.1$, while Type $\mathrm{Ib} / \mathrm{II}$ are bluer than these values. This distribution seems to reflect the distribution of various SN Types in different regions of the parent galaxies and in different galaxies as well. SNe II/Ib likely derive from massive progenitors in spiral galaxies and thus are associated to star-formation regions where a strong $\mathrm{H} \alpha$ emission is present (Van Dyk et al. 1996 and references therein). Type Ia SNe, instead, possibly derive from a wider variety of stellar environments (as they are observed both in elliptical and spiral galaxies). 
Acknowledgements. F.R.B. would like to thank Angela Bragaglia, David Branch, Enrico Cappellaro, Massimo Della Valle, Boris Dirsch, Claus Leitherer, Arturo Manchado, Ulisse Munari and Elena Pian for useful discussions and encouragement.

\section{References}

Barth A.J., Van Dyk S.D., Filippenko A.V., Leibundgut B., Richmond M.W., 1996, AJ 111, 2047

Boffi F.R., Branch D., 1995, PASP 107, 347

Branch D., 1990, in "Supernovae", Petschek A.G. (ed.). Springer-Verlag, pp. 39-42

Branch D., Livio M., Yungelson L.R., Boffi F.R., Baron E., 1995, PASP 107, 1019

Buta R.J., Williams K.L., 1995, AJ 109, 543

Cardelli J.A., Clayton G.C., Mathis J.S., 1989, ApJ 345, 245

Chevalier R.A., 1986, ApJ 308, 225

Cowan J.J., Henry R.B.C., Branch D., 1988, ApJ 329, 116

Crotts A.P.S., 1988, ApJ 333, L51

Fesen R.A., Gerardy C.L., McLin K.M., Hamilton A.J.S., 1998, astro-ph9810002

Filippenko A.V., Barth A.J., Bower G.C., et al., 1995, AJ 110, 2261

Goodrich R.W., Stringfellow G.S., Penrod G.D., Filippenko A.V., 1989, ApJ 342, 908

Lira P., et al., 1998, AJ 115, 234

Long K.S., Blair W.P., Krzeminski W., 1989, ApJ 340, L25

Longo G., de Vaucouleurs A., 1983, The University of Texas Monographs in Astronomy No. 3
Pennington R.L., Dufour R.J., 1983, ApJ 270, L7

Pennington R.L., Talbot R.J., Dufour R.J., 1982, AJ 87, 1538

Perlmutter S., et al., 1998 (in preparation)

Porter A.C., 1993, PASP 105, 1250

Riess A.G., et al., 1998, AJ 116, 1009

Schmidt B.P., Kirshner R.P., Leibundgut B., et al., 1994, ApJ 434, L19

Rieke G.H., Lebofski M.J., 1985, ApJ 288, 618

Sparks W.B., 1994, ApJ 433, 19 (Paper I)

Sparks W.B., 1996, ApJ 470, 195 (Paper II)

Sparks W.B., Paresce F., Macchetto F., 1989, ApJ 347, L65

Sparks W.B., Macchetto F., Panagia N., et al., 1999, ApJ (accepted)

Van Dyk S.D., Hamuy M., Filippenko A.V., 1996, AJ 111, 2017

de Vaucouleurs G., de Vaucouleurs A., Corwin H.G., et al., 1986, "Third Reference Catalog" (RC3)

de Vaucouleurs A., Longo G., 1988, The University of Texas Monographs in Astronomy, No. 5

Weiler K.W., van der Hulst J.M., Sramek R.A., Panagia N., 1981, ApJ 243, L151

Weiler K.W., Sramek R.A., Panagia N., van der Hulst J.M., Salvati M., 1986, ApJ 301, 790

Weiler K.W., Van Dyk S.D., Panagia N., Sramek R.A., Discenna J.L., 1991, ApJ 380, 161

Zombek M.V., 1992, Handbook of Space Astronomy and Astrophysics. Cambridge University Press, Chapter 2, p. 68

Zwicky F., 1965, in "Stars and Stellar Systems", Vol. VIII (Stellar Structure), Aller L.H. and McLaughlin D.B. (eds.). Chicago: University of Chicago Press, p. 367 